Draft VERSiOn JANUARY 1, 2019

Typeset using $\mathrm{LAT}_{\mathrm{E}} \mathrm{X}$ preprint2 style in AASTeX62

\title{
DETAILED X-RAY MAPPING OF THE SHOCKED EJECTA AND CIRCUMSTELLAR MEDIUM IN THE GALACTIC CORE-COLLAPSE SUPERNOVA REMNANT G292.0+1.8
}

\author{
Jayant Bhalerao, ${ }^{1}$ Sangwook Park, ${ }^{1}$ Andrew Schenck, ${ }^{1}$ Seth Post,${ }^{1}$ and John P. Hughes ${ }^{2}$ \\ ${ }^{1}$ Department of Physics, University of Texas at Arlington, P.O. Box 19059, Arlington, TX 76019, USA \\ ${ }^{2}$ Department of Physics and Astronomy, Rutgers University, 136 Frelinghuysen Road, Piscataway, NJ 08854-8019, \\ USA
}

(Accepted December 26, 2018, for publication in ApJ)

\begin{abstract}
G292.0+1.8 (G292) is a young ( 3000 yr), Galactic textbook-type core-collapse supernova remnant (CCSNR). It is characterized by X-ray, optical and infrared emission from ejecta and circumstellar medium (CSM) features, and contains a pulsar (PSR J1124-5916) and pulsar wind nebula that have been observed in X-rays and radio. Previous studies have revealed a complex, dynamically evolving, oxygen-rich remnant, a striking relic from the explosion of a massive star. Here, using our deep (530 ks) Chandra ACIS data, we present high spatial-resolution maps (based on a regional grid size of a few arcsec) of the shocked CSM and metal-rich ejecta in G292. We make the first Chandra-detection of Fe-rich ejecta in G292. We identify the X-ray counterpart of the northern equatorial belt, a component of a ring-like CSM structure identified earlier in the infrared band. We show the detailed spatial distributions of ejecta enriched in $\mathrm{O}$, $\mathrm{Ne}, \mathrm{Mg}, \mathrm{Si}, \mathrm{S}$ and Fe. We find that the bulk of the Si, S and Fe-rich X-ray-emitting ejecta are located in the northwestern hemisphere of the remnant, opposite to the pulsar's projected angular displacement to the southeast from the SNR's center. This suggests that the pulsar's kick may have originated from gravitational and hydrodynamic forces during an asymmetric explosion, rather than from anisotropic neutrino emission. Based on abundance ratios and our estimated CSM and ejecta masses, we constrain the progenitor mass to $13 \mathrm{M}_{\odot} \lesssim \mathrm{M} \lesssim 30 \mathrm{M}_{\odot}$
\end{abstract}

Keywords: ISM: individual objects (G292.0+1.8), structure, supernova remnants — methods: observational - X-rays: ISM

\section{INTRODUCTION}

Oxygen, a key component of life, is produced by nuclear fusion in the cores of massive stars and released into the interstellar medium when these stars explode as supernovae $(\mathrm{SNe})$.

jayant.bhalerao@mavs.uta.edu
G292.0+1.8 (G292 hereafter) is one of three known Galactic oxygen-rich core-collapse supernova remnants (CCSNR, Goss et al. 1979). The other two in this group are Cassiopeia A (Cas A) and Puppis A (e.g., Ghavamian et al. 2005 and references therein). These remnants show optical emission dominated by forbidden 
lines of oxygen from high-speed SN ejecta. The oxygen is produced by nuclear burning processes in the interiors of the massive progenitor stars that gave rise to these remnants. Oxygen and other nucleosynthesis products are expelled into interstellar space as SN ejecta when these stars explode as CCSN. Among the three known Galactic oxygen-rich remnants, only G292 shows all the textbook-type features of a CCSNR: metal-rich ejecta, shocked circumstellar medium (CSM), a rotation-powered neutron star (NS or pulsar, PSR J1124-5916) and pulsar wind nebula $(\mathrm{PWN})$ detected both in X-rays and in radio (Hughes et al. 2001, 2003; Camilo et al. 2002; Gaensler \& Wallace 2003). In contrast, Puppis A is dominated by emission from shocked gas with a low-abundant CSM and/or ISM-like composition (Hwang et al. 2008; Katsuda et al. 2008; Luna et al. 2016). The NS in Puppis A has a weak magnetic field and is radio-quiet (Gotthelf \& Halpern 2009). Cas A differs in having ejecta that are unusually low in $\mathrm{Ne}$ and $\mathrm{Mg}$ abundance, and are dominated by $\mathrm{Si}, \mathrm{S}$ and $\mathrm{Fe}$ (Vink et al. 1996; Dewey et al. 2007). The NS in Cas A does not pulsate and it is not surrounded by a PWN (e.g., Pavlov et al. 2000; Vink 2008). G292's textbook features make it very useful for studying the evolutionary processes of stars, massive-enough to eventually explode as "standard" CCSNe and create normal pulsars.

G292 is the result of an unrecorded SN explosion in an area of the southern sky marked by the bright constellation Centaurus. Its age of $\sim 3000$ yr was estimated from the observed expansion rates of fast-moving optical ejecta knots in the optical band (Ghavamian et al. 2005; Winkler et al. 2009) and from the Sedov interpretation of its X-ray shell emission spectrum (Gonzalez \& Safi-Harb 2003). This age estimate is consistent with the characteristic spindown age of G292's pulsar (2900 yr, Camilo et al. 2002). The distance to G292 has been esti- mated to be $\gtrsim 6 \mathrm{kpc}$ using $\mathrm{H}$ I absorption measurements (Gaensler \& Wallace 2003). G292 has an angular size of $\sim 9^{\prime}$ in X-ray (Park et al. 2007) and in radio (Gaensler \& Wallace 2003), which corresponds to a diameter of $\sim 16 \mathrm{pc}$ at $d=6 \mathrm{kpc}$.

At X-ray wavelengths, G292 exhibits a rich, and intricate pattern of ejecta and CSM structures. The ejecta form networks of knots and filaments distributed over the face of the entire remnant. Superimposed on the ejecta is the shocked CSM, which manifests as several distinctive morphological structures in X-rays: the equatorial belt, thin circumferential filaments, and the outermost diffuse, spectrally soft emission (Gonzalez \& Safi-Harb 2003; Park et al. 2002, 2004, 2007). The equatorial belt is a dense, belt-like feature running along the SNR's "equator." It has been observed in the optical band (Ghavamian et al. 2005), in infrared (Lee et al. 2009; Ghavamian et al. 2012; Ghavamian \& Williams 2016), and in X-rays (e.g., Tuohy et al. 1982; Hughes et al. 2001; Park et al. 2002, 2004, 2007). The thin circumferential filaments form narrow arcs of spectrally soft emission along the outer boundary of the SNR (Gonzalez \& Safi-Harb 2003; Park et al. 2002, 2007). Finally, the shocked CSM forms a diffuse, spectrally soft border along the outer edge of the SNR which marks the region where the forward shock (FS) is interacting with the red supergiant (RSG) winds of the progenitor (Park et al. 2007; Lee et al. 2010). The suggested origins for these CSM emission features may include relic structures from the outer atmosphere of a rotating progenitor star, and/or from binary interactions (e.g., Chevalier 1992; Chita et al. 2008; Morris \& Podsiadlowski 2009; Smith et al. 2013).

The ejecta in G292 are characterized by high velocity $\left(v_{\text {radial }} \gtrsim 1000 \mathrm{~km} \mathrm{~s}^{-1}\right)$ knots and filaments, also known as fast-moving knots or FMKs (Ghavamian et al. 2005; Bhalerao et. 
al. 2015). The bulk of the shocked metalrich ejecta in G292 is dominated by O, Ne and Mg (e.g., Park et al. 2004). Relatively weak Fe K-shell line emission from the Fe-rich ejecta gas has been detected in Suzaku data (Kamitsukasa et al. 2014; Yamaguchi et al. 2014), however the poor angular resolution of Suzaku $\left(\sim 2^{\prime}\right.$ HPD, Mitsuda et al. 2007) hindered its accurate spatial mapping. The location of the reverse shock $(\mathrm{RS})$ close to the outer edge of the PWN in G292 (Gaensler \& Wallace 2003; Bhalerao et. al. 2015) suggests that the interaction of the RS with the central ejecta may have recently started, therefore the bulk of the innermost ejecta, representing explosive nucleosynthesis products such as Fe, may not have been heated by the RS yet. Unlike Cas A, significant mixing and overturning of ejecta in G292 may not have occurred (Park et al. 2004; Ghavamian et al. 2012).

Previous studies have sampled a limited number of ejecta regions using Chandra data (Gonzalez \& Safi-Harb 2003; Park et al. 2004, 2007). More extensive regions of G292 have been analyzed using a regional grid (Yang et al. 2014), however, this study was based on Chandra Advanced CCD Imaging Spectrometer-S3 (ACIS-S3) data with incomplete coverage of the SNR because of the detector's smaller field of view $\left(8.3^{\prime} \times 8.3^{\prime}\right.$, Weisskopf et al. 2005). In this paper, we use data collected with the Chandra ACIS-I array, which has a larger field of view $\left(17^{\prime} \times 17^{\prime}\right)$ and covers the entire SNR. Furthermore, the ACIS-I data we use here has an order of magnitude longer exposure than the ACIS-S3 data.

Evidence for a strong link between asymmetric SN explosions and "NS-kicks" (forces imparted to the NS during the SN explosion) is emerging (e.g., Janka 2017 and references therein). In G292, evidence for an asymmetric explosion has been suggested. Si-rich ejecta gas is observed mainly in the north and north- west of the SNR (Park et al. 2002; Yang et al. 2014). Radial velocity measurements detect a significantly larger number of blueshifted knots compared to redshifted ones (Ghavamian et al. 2005; Bhalerao et. al. 2015). Also, the radial velocity magnitudes of the blueshifted knots are generally higher than those of the redshifted ones (Bhalerao et. al. 2015). Oxygenrich ejecta filaments in the optical band show higher proper motions in the north-south direction than in the east-west direction (Winkler et al. 2009).

The detailed distribution of Fe-rich ejecta in G292 is unknown. Fe is a key explosive nucleosynthesis product, produced in the deepest layers of the SN, and its spatial distribution, especially asymmetric patterns, would be crucial for revealing the nature of the CCSN explosion (Woosley et al. 2002; Thielemann et al. 2007; Maoz \& Graur 2017).

Here, using our deep Chandra ACIS-I data, we perform a detailed spectroscopic analysis of the entire remnant. This study is a direct expansion of our earlier studies of G292 based on the same Chandra data (Park et al. 2007; Lee et al. 2010). In this paper, we reveal G292's structure, morphology and distribution of the CSM and ejecta in unprecedented detail. For the first time, we provide a high-spatial-resolution map revealing the distribution of Fe-rich ejecta in G292. We also provide spatial distribution maps for O-, Ne-, Mg-, Si- and S-rich ejecta, and their thermodynamic parameters including the electron temperature and ionization timescale. We discuss these results in the context of recent CCSN hydrodynamic models, and their implications in understanding the nature of CCSN explosions. 


\section{OBSERVATIONS \& DATA REDUCTION}

Our G292 observation was performed between 2006 September 13 and 2006 October 20 using the ACIS-I array. We used the ACIS-I array since it has a large field of view $\left(17^{\prime} \times 17^{\prime}\right.$, Weisskopf et al. 2005) and can cover the entire SNR (angular size $\sim 9^{\prime}$, Park et al. 2007). The observation consisted of six individual ObsIDs with exposure times ranging from $\sim 40 \mathrm{ks}$ to 160 ks (Table 1). The aim point was close to the position of the pulsar (PSR J1124-5916) at R.A. $($ J2000.0 $)=11^{h} 24^{m} 39^{s} .1$, decl. $($ J2000 $)=$ $-59^{\circ} 16^{\prime} 20^{\prime \prime}$ (Camilo et al. 2002; Hughes et al. 2003). We processed the data with standard data reduction methods for grade and hot pixel filtering using the Chandra Interactive Analysis of Observations (CIAO, Fruscione et al. 2006), version 4.7 , with calibration database CALDB 4.6.7 . No significant background flaring was observed. The processed data had a total effective exposure time of $\sim 509 \mathrm{ks}$. We note that after we had completed our analysis for this work using our 2006 observation, additional ACIS-I data of G292 (with a total exposure of $~ 300 \mathrm{ks}$, taken in 2016) became available in the archive. Considering the time-dependent quantum efficiency degradation of the ACIS-I detector, we estimate that although these additional data would increase the total number of photons by $\sim 40 \%$, the added signal-to-noise will not alter our scientific conclusions. Thus, we do not include these additional Chandra archival data of G292 in this work.

\section{ANALYSIS \& RESULTS}

\subsection{Characterization of the Outermost Shocked CSM}

We divided the remnant into a sub-regional grid using our adaptive mesh method (Schenck et al. 2016). This technique adaptively divides the SNR into small rectangular sub-regions, to contain a certain minimum number of counts per sub-region. We used the $0.3-8 \mathrm{keV}$ band image of G292 to apply this method. To perform a statistically significant spectral analysis of each regional spectrum, we set each individual region to contain at least 5000 counts in the $0.3-8 \mathrm{keV}$ band. We find that several thousand counts are generally required to obtain measurements of spectral model parameters, including elemental abundances, to within $\sim 50 \%$ uncertainties. Constrained measurements with uncertainties $\lesssim 50 \%$ allow reliable characterization of the physical and chemical properties of the X-ray emitting gas in SNRs. From our initial spectral analysis of a small number of regions in G292, we found that 5000 counts were required for such measurements. Our adaptive mesh results in 2147 regions (Fig. 1a). The sizes of the regions range from $\sim 2^{\prime \prime} \times 4^{\prime \prime}$ for regions enclosing the bright filaments, to $\sim 30^{\prime \prime} \times 50^{\prime \prime}$ for regions representing the faint outer boundary of the SNR. The average angular area of the regions is $\sim 115 \operatorname{arcsec}^{2}$.

To estimate mean elemental abundances in the shocked CSM in G292, we extracted X-ray spectra from several representative CSM emission regions identified in previous works (e.g., Lee et al. 2010; the spectrally-soft red diffuse regions near the outermost boundary of the SNR in Fig. 1b). We fitted these regional spectra using the absorbed non-equilibrium ionization (NEI) plane-parallel shock model (Borkowski et al. 2001) with varied abundances (vpshock, NEI version 3.0 with updated ATOMDB (Smith et al. 2001; Badenes et al. 2006; Foster et al. 2012) in XSPEC (Arnaud 1996) and ISIS, (Houck \& Denicola 2000). We varied the foreground column $\left(N_{H}\right)$, normalization, electron temperature $(k T$, where $k$ is the Boltzmann constant and $T$ is the temperature), ionization timescale $\left(n_{e} t\right.$, where $n_{e}$ is the postshock electron number density and $t$ is the time elapsed since the passage of the shock), and elemental abundances for $\mathrm{O}, \mathrm{Ne}$, $\mathrm{Mg}, \mathrm{Si}, \mathrm{S}$ and $\mathrm{Fe}$. We fixed the abundances 
of all other elements to solar values (Anders \& Grevesse 1989). The measured abundances and thermodynamic parameters did not show significant variation among the regions (within statistical uncertainties), therefore we took the average values to represent the CSM emission. These average values are consistent with previous estimates (Lee et al. 2010) and are listed in Table 2.

\subsection{Origin of the Regional Emission (Ejecta vs. CSM)}

To characterize the emission across the remnant, we extracted source emission spectra from all 2147 regions. We subtracted the background emission using spectra extracted from sourcefree regions outside the remnant. We used a total of four circular background regions located beyond the outer boundary of the SNR, with one background region located on each of the four CCDs of the ACIS-I detector. We combined these four regions to characterize the "average" background spectrum. We fit spectra for all the individual regions with an NEI plane shock model. For regions projected within or near the PWN, the contribution in the observed regional spectrum from the nonthermal synchrotron radiation from the PWN may be significant. Therefore, we added a power-law component in our spectral model fits for $\sim 310$ regional spectra generally projected against the spectrally-hard central PWN feature (Hughes et al. 2001; Park et al. 2007). The locations of these regions are outlined with a blue dashedcurve in Fig. 1c. For our spectral model fits, the parameters we varied are: $N_{H}$, normalization, $k T, n_{e} t$ and the abundances for $\mathrm{O}, \mathrm{Ne}, \mathrm{Mg}, \mathrm{Si}$, $\mathrm{S}$ and Fe. We fixed the abundances of all other elements to solar values (Anders \& Grevesse 1989).

We classified regions as ejecta-dominated if the measured abundance for any fitted elemental abundance exceeded our measured CSM abundance values (listed in Table 2) by more than a $3 \sigma$ confidence level. Using this method, we identified $\sim 1400$ ejecta-dominated regions (white regions in Fig. 1c), and $\sim 700$ CSMdominated regions (brown regions in Fig. 1c). In the central regions of the SNR, 150 CSMdominated regions and $\sim 160$ ejecta-dominated regions are affected by synchrotron continuum emission from the PWN (these regions are outlined by a blue dashed-curve in Fig. 1c). These regions show a relatively high continuum flux in the $2-7 \mathrm{keV}$ band $(\sim 10 \%-60 \%$ of the total flux). They yielded fits with $\chi^{2} / \nu>2$ and/or unrealistically high electron temperature $(k T \sim$ 3-10 keV) when no power-law component was included in the spectral model. After we added the power-law component, these PWN regions yielded improved fits with $\chi^{2} / \nu \sim 0.9$ to 1.5 , and $k T \lesssim 0.7 \mathrm{keV}$. The best-fit photon index is $\Gamma \sim 1-3$ for these PWN regions, which is generally consistent with values estimated for G292's PWN regions in the literature (Gonzalez \& SafiHarb 2003). Overall, our approach yields statistically acceptable fits $\left(\chi^{2} / \nu<2\right)$ for over $99 \%$ of the 2147 regions comprising the SNR (Fig. 5a). In Fig. 2, we show an example spectrum of an ejecta-dominated region (region " $\mathrm{E}$ " in Figs. 1a \& 1c), and a CSM-dominated region (region "C" in Figs. 1a \& 1c). Both spectra in Fig. 2 were fitted with an NEI plane shock model assuming our measured average CSM abundances (Table 2). The ejecta-dominated region gives a poor fit to this model $\left(\chi^{2} / \nu=6.6\right)$ due to excessive line fluxes originating from overabundant ejecta gas (primarily from the Ne K and $\mathrm{Mg} \mathrm{K}$ lines at $E \sim 1.0 \mathrm{keV}$ and $\sim 1.35 \mathrm{keV}$ respectively). The CSM-dominated region, on the other hand, gives a good fit $\left(\chi^{2} / \nu=1.2\right)$.

\subsection{Spatial Distribution of the Elemental Abundances and NEI Plasma Parameters}

We show the measured abundance distributions of $\mathrm{O}, \mathrm{Ne}, \mathrm{Mg}, \mathrm{Si}, \mathrm{S}$ and Fe for the entire remnant in Fig. 3. For comparisons, we also show atomic line equivalent width image (EWI) 
maps for these elements in Fig. 3. We position these maps next to each other to allow for easier side-by-side, element by element, comparisons of regional abundances and emission line strengths. We created the EWI maps using published methods (e.g., Hwang et al. 2000; Park et al. 2002; Schenck et al. 2014). The line and continuum band energies used for making these maps are listed in Table 3 . We binned the images by $2 \times 2$ pixels, and adaptively smoothed them before performing the EWI calculations. These EWI maps are updated versions of those we had published earlier (Park et al. 2002). Our previous EWI maps were based on $\sim 43 \mathrm{ks}$ of Chandra data taken on the ACIS-S3 wherein parts of the remnant were not imaged due to the small field of view of the ACIS-S3. Our new EWI maps utilize over an order of magnitude higher count statistics than our previous maps. These higher photon counts allow us to rebin the subband images with a significantly smaller number of pixels in our new EWI maps, as compared to our previous maps. This binning advantage helps in effectively creating a higher spatial resolution in our new EWI maps. Our new EWI maps also cover the entire SNR. In this work, we provide EWI maps that have not been published before, namely those for $\mathrm{Mg}$ XII, S XV and Fe K (Fe He $\alpha$ or Fe XXV). In our EWI maps, regions with strongly enhanced EWs are generally coincident with highly overabundant regions in our elemental abundance maps (Fig. 3), indicating that the strong line fluxes are primarily caused by the presence of overabundant ejecta gas. In Fig. 3, we label the EWI maps based on the most prominent X-ray emission lines observed for SNR plasmas (e.g., Vink 2017).

In Fig. 4, we show abundance-ratio maps (for the ejecta-dominated regions) of $\mathrm{O} /(\mathrm{Si}+\mathrm{S}+\mathrm{Fe})$, $\mathrm{Ne} /(\mathrm{Si}+\mathrm{S}+\mathrm{Fe}), \mathrm{Mg} /(\mathrm{Si}+\mathrm{S}+\mathrm{Fe}), \mathrm{Si} /(\mathrm{O}+\mathrm{Ne}+\mathrm{Mg})$, $\mathrm{S} /(\mathrm{O}+\mathrm{Ne}+\mathrm{Mg})$ and $\mathrm{Fe} /(\mathrm{O}+\mathrm{Ne}+\mathrm{Mg})$ that highlight the enhancements of lighter O-group ele- ments and the heavier $\mathrm{Si}, \mathrm{S}$ and $\mathrm{Fe}$ relative to each other. Figs. $3 \mathrm{i}-\mathrm{n}$ and Figs. $4 \mathrm{~d}-\mathrm{f}$ illustrate the anti-alignment between the pulsar and the heavier Si-, S- and Fe-rich ejecta, with the heavier ejecta clearly localized in the northnorthwestern regions of the SNR opposite to the projected location of the pulsar (see Section 4.4).

In Fig. 5, we show the spatial distributions of the NEI spectral parameters: $N_{H}, k T, n_{e} t$, the pseudo electron density $\left(n_{E M}\right)$, and the pseudo thermal pressure $\left(P_{E M}\right)$. We calculated the pseudo density as $n_{E M}=\sqrt{E M / V}$, where $E M$ is the volume emission measure and $V$ is the emission volume (described in Section 4.2). We calculated the pseudo thermal pressure as $P_{E M}=n_{E M} k T$. We label these quantities as "pseudo" since they are derived from the projected density and temperature of the SNR gas, and not the actual 3D distributions of these quantities within the remnant.

To reveal the detailed radial structure in the ionization state and ejecta abundances, we constructed radial profiles for $k T, n_{e} t$, and the abundances of $\mathrm{O}, \mathrm{Ne}, \mathrm{Mg}, \mathrm{Si}, \mathrm{S}$ and Fe along the NW and SE directions. To effectively study such radial structures, we selected the NW sector of the SNR, where the layering structure of abundances and the progressively ionized nature of the ejecta along the radial distance from the SNR center have been suggested (Park et al. 2002). For comparisons, we also performed a similar study in the opposite area of the SNR (the SE region). This "axis" along NW-SE is also intriguing in light of its alignment with the projected pulsar kick direction (Figs. 3, 4, Section 4.4). For these radial profiles, we divided the ejecta regions into radial intervals of $\sim 15^{\prime \prime}$. We show these regions in Fig. 6a, and the radial profiles in Figs. 6b-i.

\subsection{Fe-Rich Emission Regions}

We note that the Fe-abundances (Fig. 3k) were measured primarily based on the Fe L- 
line complex $(E \sim 0.7-1.2 \mathrm{keV})$, since the $\mathrm{Fe}$ K-shell line (at $E \sim 6.6 \mathrm{keV}$ ) is weak or undetectable in most individual regional spectra. We fitted the spectra extracted from Fe-rich regions, with a plane-parallel shock model, with the Fe-abundance fixed at our measured average CSM value $(0.13 \times$ solar, Table 2$)$, while varying the abundances of $\mathrm{O}, \mathrm{Ne}, \mathrm{Mg}, \mathrm{Si}, \mathrm{S}, \mathrm{Ar}$ and $\mathrm{Ca}$. The abundance of $\mathrm{Ni}$ was tied to Fe. We varied the $N_{H}$, normalization, $k T$ and $n_{e} t$. These model fits resulted in strong residuals at $E \sim 1.2 \mathrm{keV}$, consistent with excess emission from the Fe L complex (e.g., Vink 2012; Kamitsukasa et al. 2014 and references therein). We show an example spectrum with model fits for such a region (Region A in Fig. 7) in Figs. 8a \& b. For comparisons, we show an example spectrum of an ejecta region with low Fe abundance, which shows no significant residuals relative to the best-fit model at $E \sim 1.2 \mathrm{keV}$ (Region B in Fig. 7) in Figs. 8c \& d.

The Fe-enhancements are supported by our detection of $\mathrm{Fe} \mathrm{K}$-shell line in the integrated spectrum of larger areas over the Fe-enhanced regions with significantly higher photon statistics $\left(\sim 10^{6}\right.$ counts in the $0.3-8.0 \mathrm{keV}$ band $)$. We show these large regions in Fig. 7 (regions 1 and 2) and their spectrum in Fig. 9a. For comparisons, the spectrum of an "Fe-poor" region, (region 3 in Fig. 7 , containing $\sim 1.7 \times 10^{6}$ counts in the $0.3-8 \mathrm{keV}$ band) shows significantly weaker Fe K-shell line emission (Fig. 9b). We estimate a $6 \sigma$ confidence level detection of the Fe K-shell line in the Fe-rich region, while the Fe K-shell line detection in the Fe-poor region's spectrum is marginal (at a $3 \sigma$ confidence level).

We measured the line center energy for the observed Fe K-shell line in G292 by Gaussianfitting of the spectrum extracted from combining $\sim 30$ Fe-overabundant northern regions. We varied the line center energy, line width and normalization in the Gaussian model. We measure a line center energy of $6.62 \pm 0.08 \mathrm{keV}$ (90\% con- fidence range) for the Fe K-shell emission line (Fig. 10). Our measured Fe K-shell line center energy agrees with earlier Suzaku measurements (Kamitsukasa et al. 2014; Yamaguchi et al. 2014), and is consistent with the detected values for other CCSNRs (Yamaguchi et al. 2014).

\section{DISCUSSION}

\subsection{Spatial Structure of Ejecta Elements and NEI Plasma Parameters}

The ejecta in G292 show striking differences between the spatial distributions of $\mathrm{O}, \mathrm{Ne}$ and $\mathrm{Mg}$ (representing hydrostatic nucleosynthesis products), and $\mathrm{Si}, \mathrm{S}$ and $\mathrm{Fe}$ (tracers for explosive nucleosynthesis, especially $\mathrm{Fe}$ ). While O-, Ne-, and Mg-rich ejecta are widely scattered across the remnant, they appear to be particularly enhanced in the NW and SE quadrants (Figs. 3a-c). This preferred distribution is brought out in our $\mathrm{O} /(\mathrm{Si}+\mathrm{S}+\mathrm{Fe})$, $\mathrm{Ne} /(\mathrm{Si}+\mathrm{S}+\mathrm{Fe})$ and $\mathrm{Mg} /(\mathrm{Si}+\mathrm{S}+\mathrm{Fe})$ abundance ratio maps (Figs $4 \mathrm{a}-\mathrm{c}$ ). In contrast to the abundances of the O-group elements, the abundances of the heavier elements, Si, S and Fe, are enhanced almost exclusively in the NW regions (Figs. 3i-n). This enhancement is also revealed in our $\mathrm{Si} /(\mathrm{O}+\mathrm{Ne}+\mathrm{Mg}), \mathrm{S} /(\mathrm{O}+\mathrm{Ne}+\mathrm{Mg})$ and $\mathrm{Fe} /(\mathrm{O}+\mathrm{Ne}+\mathrm{Mg})$ abundance ratio maps (Figs. $4 d-f)$.

X-ray line emission for $\mathrm{O}, \mathrm{Ne}, \mathrm{Mg}, \mathrm{Si}, \mathrm{S}$ and $\mathrm{Fe}$, shown in our line EW maps, reveals distributions that are generally consistent with our abundance maps (Fig. 3). A similarity between the spatial distribution patterns for the line EWs for O, Ne IX and Mg XI is observed (Figs. 3d-f). These line EWs are generally enhanced close to the projected position of the RS. $^{1}$

\footnotetext{
${ }^{1}$ The projected position of the RS at $r_{R S} \sim 130^{\prime \prime}$ and $\mathrm{CD}$ at $r_{C D} \sim 220^{\prime \prime}$ are $1 \mathrm{D}$ approximations, estimated based on G292's kinematic structure in X-rays (Bhalerao et. al. 2015). $r$ is the angular radius measured from the optical expansion center of the remnant at RA (2000.)
} 
In the SE, the line EWs for O, Ne IX and $\mathrm{Mg}$ XI are enhanced in an area that is coincident with the optical "spur" (Ghavamian et al. 2005). The spur is a crescent-shaped structure dominated by low velocity $(\mathrm{v} \sim 100 \mathrm{~km}$ $\mathrm{s}^{-1}$, Ghavamian et al. 2005), dense, clumpy ejecta that are radiatively cooling to emit in the optical band (e.g., [O III], Ghavamian et al. 2005; Winkler \& Long 2006) and in infrared (e.g., [O IV], Ghavamian et al. 2012). While the optical and X-ray emitting ejecta in the SE may not precisely share the same spatial locations throughout the remnant, probably some of the low-density, high velocity X-ray ejecta have mixed with the higher density optical ejecta to produce the observed X-ray emission of O, Ne IX, and Mg XI from the southeastern regions of the SNR. Similar spatial correlations between optical and X-ray emission in G292 have been observed before (Park et al. 2002, 2007; Ghavamian et al. 2005; Bhalerao et. al. 2015).

Line emission for Ne X and Mg XII, on the other hand, is not evident in the SE, and it peaks at larger radial distances in other parts of the remnant, closer to the contact discontinuity (CD) and even extending to the outer boundary in some areas (Figs. 3g \& 3h). This radially "layered" emission pattern is particularly emphasized in the NW. These high EW-regions in the NW are spatially coincident (in projection) with overabundant ejecta gas (Fig. 3). A similarly layered EW distribution for Ne IX and Ne X lines in the NW regions of G292 have been reported in previous work (Park et al. 2002). Based on our extensive spectral analysis, we show radially increasing ionization timescale values for the shocked ejecta gas (from the RS to the $\mathrm{CD}$ ) in the NW (Fig. 6c). This ra-

$=11^{h}, 24^{m}, 34^{s} .4$, and decl. $(2000)=.-59^{\circ}, 15^{\prime}$, $51^{\prime \prime}$ (Winkler et al. 2009). The actual 3D positions of the RS and CD could differ in different parts of the remnant. dial distribution of the ionization timescale supports the suggested progressive ionization of the ejecta gas by the RS on its migration towards the center of the remnant. A similar progressive ionization structure for O- and Ne-rich ejecta gas was observed in the young (age $\sim 1000 \mathrm{yr}$ ) O-rich SNR 1E 0102.2-7219 (Gaetz et al. 2000; Flanagan et al. 2004; Alan et al. 2018). The weak EWs for the highly-ionized $\mathrm{Ne} \mathrm{X}$ and $\mathrm{Mg}$ XII in the SE (Figs. $3 g$ \& $3 \mathrm{~h}$ ) are consistent with the generally lower values of the ionization timescale there $\left(n_{e} t \sim 5 \times 10^{11} \mathrm{~cm}^{-3} \mathrm{~s}\right)$ compared to those in the NW $\left(n_{e} t \sim 10^{12} \mathrm{~cm}^{-3} \mathrm{~s}\right)$. Regions in the NW located just within our identified CD show the highest $n_{e} t$. These high $n_{e} t$ regions are followed by regions where $n_{e} t$ decreases radially inwards towards the RS (Figs. 5d \& 6c).

In our radial profiles, $\mathrm{O}, \mathrm{Ne}$ and $\mathrm{Mg}$ peak closer to the CD at about an angular distance of $170^{\prime \prime}-200^{\prime \prime}$ from the center of the SNR. On the other hand, Si, S and Fe abundances peak at a smaller radial distance of about $130^{\prime \prime}-150^{\prime \prime}$ from the SNR's center (Figs. 6d-6i). This provides further evidence for a layered ejecta-structure in the NW, where the classical "onion-shell" nucleosynthesis-configuration at the core of the massive progenitor appears to have been preserved in the SNR. However, this layering of the ejecta elements is not clearly evident in other directions. For example, abundances for $\mathrm{O}$ and Fe peak at about the same radial distance $(r$ $\sim 130^{\prime \prime}$ ) in the SE (blue curves in Figs. 6d-i). This may suggest some mixing of the ejecta in the SE and/or projection effects caused by Orich regions that are physically located at larger radial distances than the Fe-rich regions, but are projected closer to the SNR center along the line of sight.

Our electron temperature map shows a general range between 0.5 to $1 \mathrm{keV}$ across the remnant (Fig. 5c), but there are regions in the N and NW with significantly higher electron temperature $(k T \sim 2-4 \mathrm{keV})$. These high- 
temperature regions are also enriched in Fe (Fig. $3 \mathrm{k})$. The hot gas temperature in this region may have helped us effectively detect line emission from Si, S and Fe (especially the Fe K-shell line). The higher electron temperatures in the $\mathrm{N}$ and NW are generally consistent with the results reported by Park et al. (2007).

The absorbing column $\left(N_{H}\right)$ does not show significant variations across the remnant (Fig. $5 \mathrm{~b}$ ). The average column density over the entire remnant is $6 \times 10^{21} \mathrm{~cm}^{-2}$. There are a few regions in the $\mathrm{S}$ and SW (near the outermost boundary of the SNR) with higher $N_{H}$ $\left(\sim 8 \times 10^{21} \mathrm{~cm}^{-2}\right)$. Although the higher $N_{H}$ in the $\mathrm{S}$ and $\mathrm{SW}$ is not statistically significant, an excess $N_{H}$ in this part of the SNR cannot be ruled out. This is suggested by the enhanced dust emission observed from these regions in the far-infrared (e.g., Park et al. 2007; Ghavamian \& Williams 2016). There may be superposed H II regions in the SW (as suggested by Rodgers et al. 1960), or a molecular cloud complex projected along and outside the southwestern boundary of G292 (as suggested by Ghavamian \& Williams 2016). Detailed maps and distances to these molecular cloud structures are not known, and the presence of a higher column in the $\mathrm{S}$ and $\mathrm{SW}$ will need further investigation.

\subsection{Ejecta Mass}

We estimated the ejecta masses for the individual elements based on our estimated volume emission measure (e.g., Lopez et al. 2009 and references therein):

$$
E M_{x}=n_{e} n_{x} V f
$$

where $\boldsymbol{n}_{\boldsymbol{x}}$ is the ion number density, $V$ is the volume of the region, $f$ is the X-ray emission filling factor which characterizes the clumpiness of the emission, and the subscript $x$ designates the element or ion being evaluated. To estimate $V$ we assumed a distance of $d=6 \mathrm{kpc}$ to the SNR. We assumed that the ejecta knots and filaments represent localized volumes of X-rayemitting gas, for which the line of sight depth is of the order of the angular extent of each rectangular region.

We calculated the electron number density:

$$
n_{e}=\sqrt{\frac{E M_{x} B_{x} X_{x} N_{x}}{V}}
$$

where $B_{x}$ is the best-fit abundance, $X_{x}$ is the solar number abundance and $N_{x}$ is the average number of electrons lost by a given ion. We estimated $N_{x}$ using a simple approximation for O-rich SNR gas (Lazendic et al. 2006):

$$
N_{x}= \begin{cases}Z, & \text { for } Z \leq 9 \\ Z-2, & \text { for } 10 \leq Z \leq 16 \\ Z-10, & \text { for } Z \geq 17\end{cases}
$$

where $Z$ is the atomic number of the element. Using this approximation we get the following $N_{x}: \mathrm{O}=8, \mathrm{Ne}=8, \mathrm{Mg}=10, \mathrm{Si}=12, \mathrm{~S}=14, \mathrm{Fe}$ $=16$. To calculate $N_{x}$ for Fe, we assumed that the Fe XVII ion is the dominant contributor to the Fe L complex detected at $E \sim 0.7-1.2 \mathrm{keV}$ in G292 (e.g., Kamitsukasa et al. 2014, and this work). We note that assuming the higher ionized state of Fe XXV $\left(N_{x}=24\right)$, corresponding to the observed Fe K-shell line emission at $E=6.6 \mathrm{keV}$ (e.g., Vink 2012 and references therein, Vink 2017), does not significantly alter our estimated Fe ejecta masses. The difference in estimated Fe ejecta masses assuming these different ionization states is less than $20 \%$, and within statistical uncertainties.

We calculated the mass of the element in each ejecta region:

$$
M_{x}=n_{x} A_{x} m_{u} V f
$$

where, $n_{x}=n_{e} / N_{x}, A_{x}$ is the atomic mass for the dominant isotope and $m_{u}$ is the atomic mass unit in grams $\left(1 \mathrm{amu}=1.66 \times 10^{-24} \mathrm{~g}\right)$.

We calculated the total ejecta mass for each element by summing the calculated masses for 
all ejecta regions. We list our calculated ejecta masses for $\mathrm{O}, \mathrm{Ne}, \mathrm{Mg}$, Si, S, Fe for the entire remnant, and the total ejecta mass in Table 4. We estimate a total shocked ejecta mass of $\sim 1.29_{-0.28}^{+0.49} f^{1 / 2} d_{6}^{3 / 2} \mathrm{M}_{\odot}$. In Fig. 11 we show the "quadrants" and "hemispheres" we use to investigate asymmetries in the ejecta elemental mass distributions. We list the calculated ejecta masses for these quadrants and hemispheres in Table 4.

We estimate that a total of $\sim 0.03 \pm 0.01 f^{1 / 2}$ $d_{6}^{3 / 2} \mathrm{M}_{\odot}$ of $\mathrm{Fe}$ ejecta has been shocked by the $\mathrm{RS}$ to emit X-rays. This is significantly less than the Fe-yield of $\sim 0.07-0.1 \mathrm{M}_{\odot}$ predicted for a wide range of progenitors $(\mathrm{M}=13-40$ $\left.\mathrm{M}_{\odot}\right)$, metallicity $(Z=0-0.02)$ and explosion energy $\left(E=1-30 \times 10^{51}\right.$ ergs)(Nomoto et al. 2006). Similarly, we estimate that $\sim 0.06 \pm 0.01$ $f^{1 / 2} d_{6}^{3 / 2} \mathrm{M}_{\odot}$ of Si ejecta, and $\sim 0.02 \pm 0.01 f^{1 / 2}$ $d_{6}^{3 / 2} \mathrm{M}_{\odot}$ of $\mathrm{S}$ ejecta have been shocked (Table 4). Thus, $\sim 10-80 \%$ of $\mathrm{Si}$, and $\sim 30-80 \%$ of $\mathrm{S}$ ejecta may still remain unshocked in the SNR (comparing them with yields predicted for progenitors with mass $13-40 \mathrm{M}_{\odot}, Z=0.02$ and $E=$ $1 \times 10^{51}$ ergs, Nomoto et al. 2006, also see Section 4.5). This suggests that only the outskirts of the central ejecta gas may have been shocked by the RS. This scenario is supported by the undistorted morphology of the PWN, an indication that the RS may have just reached the central regions of the SNR where the $\mathrm{Si}-, \mathrm{S}-$ and Fe-rich ejecta are expanding (Gaensler \& Wallace 2003; Gaensler \& Slane 2006; Bhalerao et. al. 2015).

\subsection{Properties of the CSM}

We identified the CSM-dominated regions in G292 based on their low abundances estimated by our regional spectral model fits. Our CSMdominated regions in Fig. 1c trace the various CSM features identified in earlier works (see Section 1). These include the thin circumferential filaments and the spectrally soft diffuse emission at the outer boundary. The origin of the thin circumferential filaments is unclear. They may be the result of the blast wave interacting with stellar wind structures produced during late evolutionary stages of the G292 progenitor (e.g. RSG and blue supergiant phases, Park et al. 2002). The "inner boundary" of the spectrally soft diffuse outermost rim of the SNR generally conforms to our estimated CD location, which marks the boundary between the CSM and ejecta (Fig. 1c). The spectrally soft emission from the CSM may also be projected over the face of the entire SNR, "filling in" the regions between the spectrally-hard ejecta filaments.

A prominent CSM feature is the central equatorial "belt" (Park et al. 2002, 2004; Ghavamian et al. 2005). Based on their multiband infrared $A K A R I$ observations, Lee et al. (2009) proposed that this feature is actually a ring-like structure that in projection presents as two long filaments, a northern filament and a southern filament, that run east-west in the central regions of the SNR (Fig. 1d). Only the bright southern filaments of this structure were previously identified in X-rays (Park et al. 2002). In Fig. 1c we show that the regions corresponding to the northern filament of the equatorial ring (ER), in addition to those for its southern filaments, are generally coincident with our identified CSM-dominated regions. These filaments appear to connect into a closed loop on the eastern side but are more fragmented on the western side. The limbbrightening expected for a pure ring structure does not appear to be present in the ER (Figs. 1b-d, 5e \& f).

The ER is composed of dense gas, and both the northern and southern filaments clearly stand out in our $n_{E M}$ and $P_{E M}$ maps (Figs. 5e $\& 5 \mathrm{f})$. This ring-like structure may have originated from pre-SN mass loss at the equator of the rotating progenitor and/or from interactions in a binary system (e.g., Collins et al. 
1999; Chevalier 2000; Morris \& Podsiadlowski 2009; Langer 2012). The northern filaments of the ER are about 2-3 times fainter than the southern filaments. This may be because the $\mathrm{ER}$ is non-uniform, and the northern filaments have a lower gas density than the southern filaments. The non-uniform nature of the ER may also supported by the the non-detection of limbbrightening that would normally be expected for a uniform, ring-like structure. Furthermore, the northern filaments are not detected in the optical band, while the southern filaments show faint zero radial velocity [O III] emission, but no clear $\mathrm{H} \alpha$ emission. (Ghavamian et al. 2005). This again suggests a non-uniform structure in the ER, with the southern filaments possibly being denser than the northern filaments, thereby demonstrating radiative cooling.

Based on the ratio of the ring's N-S angular separation $\left(\sim 75^{\prime \prime}\right)$ to its E-W diameter $\left(\sim 295^{\prime \prime}\right)$, we estimate that the normal to the ring is inclined to the plane of the sky by an angle of $\alpha \sim 15^{\circ}$. If the ER was shed by the progenitor's equatorial winds before the SN explosion, then this inclination angle would correspond to the inclination angle of the progenitor's rotation axis. This is generally consistent with the estimated N-S alignment of the spin axis of the embedded pulsar (J1124-5916) inferred from the observed N-S orientation of its jet (Park et al. 2007). This suggests that the rotation axis of the progenitor appears to have been preserved in the neutron star after the SN explosion.

We estimate the CSM mass in G292, by assuming a fully ionized plasma with $10 \% \mathrm{He}$, giving the relationship $n_{e}=1.2 n_{H}$. To estimate the volumes of the ER regions we assume the ER has a ring-like geometry with a line of sight depth similar to its N-S angular thickness $\left(\sim 15^{\prime \prime}\right)$. Based on this geometry we estimate the total volume of the $\mathrm{ER} \sim 8 \times 10^{54} f d_{6}^{3} \mathrm{~cm}^{3}$. To estimate the volume of the outer spherical CSM, we assume it has a shell-like structure with a line of sight depth similar to its angular thickness $\left(\sim 45^{\prime \prime}\right)$. For the spherical CSM projected towards the interior of the remnant, we assume a line of sight depth of $90^{\prime \prime}$ corresponding to emission from the CSM shell both at the front, and at the back of the remnant. We calculate the total CSM mass by summing the masses for all $\sim 700 \mathrm{CSM}$ regions. We list our estimates for the CSM mass in Table 5. We estimate a shocked CSM mass of $13.5_{-1.4}^{+1.7} f^{1 / 2}$ $d_{6}^{3 / 2} \mathrm{M}_{\odot}$. This is close to the lower bound of the previous estimates based on the radial density profile of the progenitor star's RSG winds $(\sim 15-$ $40 \mathrm{M}_{\odot}$, Lee et al. 2010). The ER comprises $\sim 13 \%$ of the total estimated shocked CSM mass (Table 5). This may suggest that a significant pre-SN mass loss occurred through the progenitor's equatorial winds. The ER is reminiscent of similar ring-like circumstellar features observed in SN 1987A (e.g., Frank et al. 2016 and references therein) and the B-type supergiant SBW1 (Smith et al. 2013). Such structures may originate from mass loss facilitated by the rapid rotation of the progenitor, binary interactions, confinement of the shed mass by magnetic fields, and possible combinations of these factors (e.g., Kurfurst et al. 2018 and references therein).

We estimate an average thermal pressure of $\sim 5 \times 10^{-9}$ ergs $\mathrm{cm}^{-3}$ for the CSM at the outer boundary of the remnant. This is comparable to the estimated ram pressure of the PWN $\left(2.6 \times 10^{-9} d_{6}^{-2}\right.$ ergs $\mathrm{cm}^{-3}$, Hughes et al. 2003). This may support the interaction between the RS and PWN as suggested by Bhalerao et. al. 2015. An early-stage RS-PWN interaction scenario has also been suggested by Gaensler \& Wallace (2003), based on the close juxtaposition between the PWN and the overlying shell in radio and X-rays. We note that previously it had been suggested that the RS may not have reached the PWN (Park et al. 2004). This was based on the estimation of a large pressure difference between the ER and the PWN. Our 
pseudo thermal pressure map (Fig. 5f), shows that regions in the southern filaments of the ER generally have higher gas pressures compared to other regions in the SNR. Thus the high pressure difference estimated by Park et al. (2004) may have been due to the selection of a highpressure region from the southern filaments of the ER (Region 1 in Park et al. 2004), rather than a region with typical gas pressure generally found between the FS and RS.

\subsection{Ejecta Asymmetry in the Remnant}

CCSN explosions involve a complex interaction among several physical processes, the details of which are not fully understood (e.g., Janka et al. 2012; Burrows 2013; Couch 2017). Observations indicate that a property common to many CCSNe is explosion asymmetry (e.g., Wang et al. 2001, 2003; Leonard et al. 2006; Wang \& Wheeler 2008; Lopez et al. 2011; Vink 2012; Lopez \& Fesen 2018 and references therein). Recent theoretical studies have suggested that asymmetries in CCSNe can originate from hydrodynamic instabilities during the SN explosion (Janka 2017 and references therein). These studies show that the bulk of intermediate mass elements ( $\mathrm{Si}, \mathrm{S}, \mathrm{Ar}$, $\mathrm{Ca}$ and $\mathrm{Ti}$ ) and the heavier iron-group elements ( $\mathrm{Cr}, \mathrm{Fe}, \mathrm{Ni}$ ) are ejected opposite to the direction in which the NS is kicked. A direct correlation is also found between NS-kick velocities and ejecta asymmetries (Wongwathanarat et al. 2013, 2017).

This anti-alignment or "antipodal" asymmetry between NSs and the ejecta has been observed in several CCSNRs. Analysis of Chandra and ROSAT data with the power-ratio method showed evidence for such an asymmetry in five CCSNRs: G292, CTB 109, Cas A, PKS 1209-51 and Puppis A (Holland-Ashford et al. 2017). However, in the study by Holland-Ashford et al. (2017), X-ray emission was examined in the $0.5-2.1 \mathrm{keV}$ energy range (thus emissions from $\mathrm{S}$, and the Fe K-shell transitions were not included), and a separation between emission originating from ejecta-rich regions as opposed to that from the CSM was not made. Katsuda et al. (2018a) applied an image-decomposition method to Chandra and XMM-Newton data to find that the elements Si, S, Ar and Ca were predominantly ejected opposite to the direction of NS motion in six CCSNRs: G292, Cas A, Puppis A, Kes 73, RCW 103, and N49. High-energy X-ray studies of Cas A using NuSTAR revealed that ${ }^{44} \mathrm{Ti}$, which is produced by explosive Siburning in the inner regions of the exploding star, is distributed opposite to the direction of the NS-motion (Grefenstette et al. 2014). ${ }^{44} \mathrm{Ti}$ asymmetry in the form of redshifted ${ }^{44} \mathrm{Ti}$ emission lines was also detected in SN 1987A, suggesting that its yet-undetected compact remnant may have been kicked towards the front of the remnant (Boggs et al. 2015; Wongwathanarat et al. 2017).

To investigate the ejecta spatial asymmetry in G292, we divided the ejecta regions into quadrants and hemispheres (Fig. 11). These divisions were made with reference to the optical expansion center of the SNR (RA (2000.) = $11^{h}, 24^{m}, 34^{s} .4$ and decl. (2000.) $=-59^{\circ}, 15^{\prime}$, 51", Winkler et al. 2009), and the presumed direction of the pulsar's kick to the SE (Park et al. 2007; Winkler et al. 2009). We list the ejecta masses estimated for the quadrants and hemispheres in Table 4, and we show their fractional mass distributions in Figs. 12 \& 13. We list the average abundances for the ejecta regions in the entire remnant, as well as for the ejecta regions in the quadrants and the hemispheres in Table 6.

The estimated ejecta masses of $\mathrm{Si}, \mathrm{S}$ and Fe are significantly higher in the NW hemisphere than in the SE. The NW hemisphere accounts for $\sim 90 \%$ of the total Si-ejecta mass, $\sim 100 \%$ of the total S-ejecta mass, and $\sim 60 \%$ of the total Fe-ejecta mass (Table 4, Fig. 12). A possible explanation for this non-uniform ejecta distribu- 
tion may be an asymmetric RS-structure, where the RS has not propagated deep enough through the ejecta in the SE to heat the heavier Si-, Sand Fe-rich ejecta (given a layered ejecta composition). However, the detection in the optical band, of $[\mathrm{S} \mathrm{II}]$ ejecta in the southeast (Winkler \& Long 2006), does not support this explanation. Also, in the SE, the abundances of O, Ne, $\mathrm{Mg}, \mathrm{Fe}$ and the ionization timescale are generally enhanced between $\sim 120^{\prime \prime}$ and $\sim 170^{\prime \prime}$ from the SNR center. Thus in the SE, the RS may be located at $\sim 120^{\prime \prime}$ from the SNR center, which is similar to that in other parts of the remnant. While Fe is enhanced in the NW regions of the SNR, the overall projected distribution of the Fe-rich ejecta detection over the SNR appears to be nearly circular in shape, generally coincident with the circular outline of the RS (Fig. $3 \mathrm{k})$. Thus, we propose that the apparent "lack" of heavy elements in the SE regions of G292 is probably caused by an intrinsically asymmetric distribution of Si-, S- and Fe-rich ejecta gas rather than by a significantly asymmetric RS structure.

Most of the Si, S and Fe ejecta are enhanced in regions opposite to the currently projected position of the pulsar (PSR J1124-5916, Figs. $1 \mathrm{~b} \& 3)$. The pulsar is displaced $\sim 46^{\prime \prime}$ from the presumed SN explosion center (RA (2000.) = $11^{h}, 24^{m}, 34^{s} .4$ and decl. (2000.) $=-59^{\circ}, 15^{\prime}$, 51", Winkler et al. 2009). This displacement corresponds to an average transverse velocity of $\sim 440 \mathrm{~km} \mathrm{~s}^{-1}$ at $d=6 \mathrm{kpc}$ and age $\sim 3000 \mathrm{yr}$ (Hughes et al. 2001, 2003; Winkler et al. 2009). This suggested motion of the pulsar is likely the result of a "kick" to the newly-formed NS during the SN explosion (Lai 2001; Park et al. 2007; Winkler et al. 2009). Recent 2D and 3D numerical simulations demonstrate that significant NS-kicks in the range of a few hundred to $\sim 1000 \mathrm{~km} \mathrm{~s}^{-1}$ can be produced by asymmetric SN explosions in which the bulk of intermediate and iron-group elements (e.g., Si and $\mathrm{Fe}$ ) are ejected in the opposite direction to the NS-kick (e.g., Wongwathanarat et al. 2013, 2017; Janka 2017 and references therein). Consequently, the NS and the bulk of the inner ejecta (the explosive nucleosynthesis products) would move in opposite directions of each other in the resulting SNR. The kick imparted to the NS mainly originates from gravitational and hydrodynamic forces exerted by the asymmetric ejecta on the NS, and from momentum conservation. Anisotropic neutrino emission plays only a minor role (Scheck et al. 2006; Wongwathanarat et al. 2013; Janka 2017). The NS is accelerated by the gravitational forces exerted by the slower, denser ejecta found in the hemisphere opposite to the stronger explosion. The acceleration of the NS can last several seconds, so that while the NS is pulled into one hemisphere, explosive nucleosynthesis of Fe-group elements proliferates in the opposite hemisphere (Scheck et al. 2006; Nordhaus et al. 2010, 2012; Wongwathanarat et al. 2013, 2017; Janka 2017).

In this study, we provide the first observational evidence that the bulk of Fe was ejected opposite to the NS's kick (e.g., Figs. 3k, 3n, 4f, $9 \mathrm{a} \& 12)$. This spatial distribution of the Ferich ejecta is in close agreement with $3 \mathrm{D}$ simulations which predict that ${ }^{56} \mathrm{Ni}$, the parentisotope of ${ }^{56} \mathrm{Fe}$, is mainly formed and ejected opposite to the NS's kick vector (Wongwathanarat et al. 2013, 2017). Along with Fe, we also show that the bulk of Si and S was ejected opposite to the NS's kick (e.g., Figs. 3, 4 \& 12). Considering the concentration of dense, cooler ejecta gas in the SE quadrant of the SNR, as observed in the optical and infrared bands (Ghavamian et al. 2005, 2009, 2012; Winkler \& Long 2006; Winkler et al. 2009), it is plausible that asymmetries in the explosion led to density variations in the ejecta. As a result, the O-, and Ne-rich ejecta expelled to the southeast are denser and clumpier than they are in other 
parts of the remnant. Thus, as suggested by numerical simulations (e.g., Wongwathanarat et al. 2013, 2017; Janka 2017), the NS may also have been gravitationally pulled to the southeast by these denser, slower moving, O-, Ne-rich ejecta. These ejecta are now radiatively cooling, and appear as the prominent crescent-shaped spur observed in the optical and infrared bands (Ghavamian et al. 2005, 2009, 2012; Winkler \& Long 2006; Winkler et al. 2009).

Our results suggest an explosion axis generally oriented NW-SE. We find that the X-rayemitting $\mathrm{O}-$, Ne- and Mg-rich ejecta are generally enhanced along the NW-SE direction (Fig. $3)$. To test the explosion kinematics projected across the plane of the sky (as suggested by this ejecta mass distribution), we estimated the O, Ne- and Mg-rich ejecta mass along the main shell of the SNR (regions between our estimated $\mathrm{RS}$ and CD, Fig. 13). We excluded regions projected within the RS for this comparison, since these ejecta may have their main kinematics along the line of sight. The combined NW+SE shell-regions account for a significantly higher fraction of the ejecta mass for $\mathrm{O}, \mathrm{Ne}$ and $\mathrm{Mg}$ ( 60\%-65\%, Fig. 13 and Table 7).

\subsection{Progenitor Mass}

To estimate the progenitor mass for G292, we compared the elemental abundance ratios for ejecta regions from the entire SNR to those predicted by CCSN nucleosynthesis models (Woosley \& Weaver 1995). In Fig. 14 we show those modeled values of elemental abundance ratios for which the progenitor masses are relatively well-discriminated. We compare these modeled ratios to our estimated mean abundance ratios for G292. Based on these comparisons, and taking into account all five plots in Fig. 14, we place an upper limit to the G292 progenitor mass of $\lesssim 30 \mathrm{M}_{\odot}$.

From our measured $\mathrm{Si} / \mathrm{Fe}$ ratio for the ejectadominated regions in G292, we obtain a progenitor mass estimate of $\sim 13-15 \quad \mathrm{M}_{\odot}$ (Fig. 14b). Our measured $\mathrm{O} / \mathrm{Fe}$ ratio for the ejectadominated regions in G292 corresponds to a progenitor mass of $\sim 15-25 \mathrm{M}_{\odot}$ (Fig. 14a). These two ratios suggest a lower limit to the G292 progenitor mass of $\gtrsim 13 \mathrm{M}_{\odot}$. Recently, Katsuda et al. (2018b) propose that both of these ratios $(\mathrm{Fe} / \mathrm{O}$ and $\mathrm{Fe} / \mathrm{Si}$ ) are more reliable indicators of the CCSN progenitor mass than the traditionally used abundance ratios of the other alpha elements to $\mathrm{Si}$ (e.g., O/Si, Ne/Si, $\mathrm{Mg} / \mathrm{Si}$, and $\mathrm{S} / \mathrm{Si}$ ). This is based on their comparisons of the progenitor mass-distributions of 41 CCSNRs in the Milky Way and the Large and Small Magellanic Clouds, to the standard Salpeter initial mass function. Based on G292's $\mathrm{Fe} / \mathrm{Si}$ ratio measured previously with Suzaku data (Kamitsukasa et al. 2014), they estimated a progenitor mass of $\lesssim 15 \mathrm{M}_{\odot}$. Our estimated lower limit is consistent with this estimate by Katsuda et al. (2018b). Thus, our abundance ratios suggest a progenitor mass in the range of $13 \mathrm{M}_{\odot} \lesssim \mathrm{M} \lesssim 30 \mathrm{M}_{\odot}$ (Fig. 14).

If we combine our calculated mass for the shocked ejecta $\left(1.29_{-0.28}^{+0.49} f^{1 / 2} d_{6}^{3 / 2} \mathrm{M}_{\odot}\right.$, Table 4), the shocked CSM $\left(13.5_{-1.4}^{+1.7} f^{1 / 2} d_{6}^{3 / 2} \mathrm{M}_{\odot}\right.$, Table 5) with a typical neutron star mass of 1.4 $\mathrm{M}_{\odot}$ and a dust mass of $0.023 \mathrm{M}_{\odot}$ estimated for G292 (Ghavamian \& Williams 2016), we obtain total mass of $\sim 16_{-1.7}^{+2.2} \mathrm{M}_{\odot}$. This mass is close to the lower bound of our estimated progenitor mass range based on the ejecta elemental abundance ratios. The progenitor mass would be higher than this calculated value depending on the mass of the unshocked ejecta, and the unshocked CSM mass lost prior to the explosion through stellar winds and binary interactions. Optically-emitting material would also add to the progenitor mass. However, the mass of the optical material may be low $(\lesssim$ a few solar masses) given that the optical emission in G292 (e.g., the integrated [O III] flux) is estimated to be about $20 \times$ less than the X-ray flux (Winkler \& Long 2006). It is not clear if the 
bulk of the $\mathrm{O}, \mathrm{Ne}$ and $\mathrm{Mg}$ ejecta have already been shocked in G292. Turbulence and mixing may have retained ejecta for these lighter elements close to the center of the explosion. There could also be reserves of unshocked Si-, S- and Fe-rich ejecta close to the SNR's center (see Section 4.2). We estimate that there could be the following amounts of unshocked ejecta in G292: $\mathrm{O} \lesssim 2.8 \mathrm{M}_{\odot}, \mathrm{Ne} \lesssim 0.4 \mathrm{M}_{\odot}, \mathrm{Mg} \lesssim$ $0.15 \mathrm{M}_{\odot}, \mathrm{Si} \lesssim 0.2 \mathrm{M}_{\odot}, \mathrm{S} \lesssim 0.1 \mathrm{M}_{\odot}$, and $\mathrm{Fe} \lesssim$ $0.07 \mathrm{M}_{\odot}$ (based on yields calculated for progenitors with mass $13-30 \mathrm{M}_{\odot}, Z=0.02$ and $E=$ $1 \times 10^{51}$ ergs, Nomoto et al. 2006). Thus there could be as much as $4 \mathrm{M}_{\odot}$ of ejecta that have not yet been shocked by the RS in G292. We note that analyses of G292's expansion dynamics have suggested similar total ejecta mass values. Analysis of G292's dynamics in the radio band has suggested a total ejecta mass of $\sim 5.9$ $\mathrm{M}_{\odot}$ (Gaensler \& Wallace 2003), and analysis of the remnant's dynamics in the X-ray band has suggested a total ejecta mass of $\lesssim 8 \mathrm{M}_{\odot}$ (Bhalerao et. al. 2015).

We compared our measured elemental ejectamass yields to those predicted for CCSN nucleosynthesis models (Nomoto et al. 2006, for $Z=$ 0.02 and $E=1 \times 10^{51}$ ergs, Fig. 15). Since our estimated total ejecta mass is only the shocked component (and thus represents a lower limit of the total ejecta mass), this comparison allows us to rule out progenitor masses with elemental ejecta yields less than our estimated values. The yields of $\mathrm{O}, \mathrm{Ne}$ and $\mathrm{Mg}$ suggest a progenitor with a mass $\gtrsim 20 \mathrm{M}_{\odot}$, with a lower limit of $\gtrsim$ $15 \mathrm{M}_{\odot}$ (Fig. 15). We note that predicted yields are model-dependent, and are sensitive to several parameters such as metallicity and rotation rate of the progenitor, and specifics of the explosion (e.g., Hirschi 2017; Fryer et al. 2018). We also note that our ejecta and CSM mass estimates may involve systematic uncertainties associated with our assumed X-ray emission volumes for individual emission regions, and the clumpiness of the emission (which is embedded in the volume filling factor $f$ ).

Theoretical calculations generally favor the production of a black hole rather than a NS for a progenitor of mass $\gtrsim 25 \mathrm{M}_{\odot}$ (e.g., Woosley et al. 2002). Exceptions to this "traditional" limit may exist; for example, recent CCSN explosion models indicate that black holes can result from progenitor explosions of $\mathrm{M} \gtrsim 15 \mathrm{M}_{\odot}$, and NSs can form from progenitors as massive as $120 \mathrm{M}_{\odot}$ (Sukhbold et al. 2016). Various factors such as the progenitor's metallicity, the presence of stellar companions, rotation rate of the progenitor, explosion energy and nucleosynthesis history can affect the outcome of the explosion. For example, a significant mass loss by a massive progenitor (with $\mathrm{M} \gtrsim 25 \mathrm{M}_{\odot}$ ) to its companion in a binary system, may lead to the formation of a NS rather than a black hole (e.g., Belczynski \& Taam 2008 and references therein). The presence of the ER in G292 suggests that its progenitor star may have been part of a binary and/or rapidly rotating (see Section 4.3). Thus if the G292 progenitor was more massive than $\sim 20-25 \mathrm{M}_{\odot}$, the formation of the observed pulsar rather than a black hole may be the result of significant mass loss of the progenitor star due to its binary interaction prior to the SN explosion. If the binary companion survived the explosion, it may be located within a radius of $\sim 70^{\prime \prime}$ from the explosion site, assuming a high runaway velocity of $\sim 500 \mathrm{~km} \mathrm{~s}^{-1}$ (e.g., Eldridge et al. 2011), and a distance to the remnant of $6 \mathrm{kpc}$. Possible contamination of the companion star's spectrum by metal-rich ejecta from G292 may also help in the search for the surviving companion (e.g., Gvaramadze et al. 2017; Hirai et al. 2018; Liu et al. 2018). From the above results of our elemental abundance ratio comparisons and ejecta yield estimates, we propose a conservative estimate of $13 \mathrm{M}_{\odot} \lesssim \mathrm{M} \lesssim 30 \mathrm{M}_{\odot}$ for the $\mathrm{G} 292$ progenitor mass. A massive progenitor $(\mathrm{M} \gtrsim$ 
$15 \mathrm{M}_{\odot}$ ), would imply an $\mathrm{O}$ or $\mathrm{B}$ main sequence star. Such a star would have produced an extended H II region around itself. Some of the diffuse, structured, optical $\mathrm{H} \alpha$ and [S II] emission observed around G292 (Winkler \& Long 2006), is probably a relic of this H II region.

\section{SUMMARY \& CONCLUSIONS}

Based on our deep Chandra data, we study the detailed structure of the shocked ejecta and CSM gas in the textbook-type supernova remnant G292.0+1.8. Our results are based on the systematic spectral analysis of over $\sim 2000$ regions covering the entire remnant. We identify the spatial distribution of Fe-rich ejecta in G292 for the first time, based on our detection of enhanced Fe L and Fe K-shell line emission. We provide spatial distribution maps for O-, Ne-, Mg- Si-, S- and Fe-rich ejecta. For the first time we identify the X-ray counterparts of the entire equatorial ring-like dense CSM, whose components had previously been detected in infrared. Based on elemental abundance ratios, and estimates of the ejecta and CSM masses, we estimate the G292 progenitor star's main sequence mass of $13-30 \mathrm{M}_{\odot}$. Our ejecta maps reveal a preferred NW-SE distribution axis for the elements $\mathrm{O}, \mathrm{Ne}, \mathrm{Mg}, \mathrm{Si}, \mathrm{S}$ and Fe. Furthermore,
Si-, S- and Fe-rich ejecta are primarily found in the northwestern hemisphere. This provides the first clear observational evidence that the inner ejecta in G292, consisting of key explosive nucleosynthesis products such as Fe, were predominantly expelled opposite to the direction of the neutron star's kick during the SN explosion. This anti-alignment between the neutron star and the heavier inner ejecta is consistent with theoretical CCSN calculations in which the neutron star-kick originates from gravitational and hydrodynamic forces exerted by the asymmetric ejecta on the NS, and from momentum conservation.

This work was supported in part by the SAO through Chandra grants GO1-12077X and GO6-7049A. JPH acknowledges support for Xray studies of supernova remnants from NASA grant NNX15AK71G. JB acknowledges support from the NASA Texas Space Grant Consortium. We thank Dr. Daniel Dewey, and Dr. Thomas G. Pannuti for valuable discussions. We also thank the MIT Chandra HETG group for useful ISIS scripts and plotting routines.

Software: CIAO (Fruscione et al. 2006), XSPEC (Arnaud 1996), ISIS (Houck \& Denicola 2000).

\section{REFERENCES}

Anders, E. \& Grevesse, N. 1989, Geochim.

Cosmochim. Acta, 53, 197

Alan, N., Park, S. \& Bilir, S. 2018, ApJ, in press (arXiv:1812.05607)

Arnaud, K. A. 1996, ASPC, 101, ed. G. H. Jacoby \& J. Barnes, 17

Badenes, C., Borkowski, K. J., Hughes, J. P., Hwang, U., \& Bravo, E. 2006, ApJ, 645, 1373

Belczynski, K. \& Taam, R. E. 2008, ApJ, 685, 400

Bhalerao, J., Park, S., Dewey, D. et al. 2015, ApJ, 800, 65

Boggs, S. E., Harrison, F. A., Miyasaka, H. et al. 2015, Science, 348, 670

Borkowski, K. J., Lyerly, W. J., \& Reynolds, S. P. 2001, ApJ, 548, 820
Burrows, A. 2013, RvMP, 85, 245

Camilo, F., Manchester, R. N., Gaensler, B. M., Lorimer, D. R., \& Sarkissian, J. 2002, ApJ, 567, L71

Chevalier, R. A. 1992, Nature, 355, 691

Chevalier, R. A. 2000, ApJ, 538, L151

Chevalier, R. A. 2005, ApJ, 619, 839

Chita, S. M., Langer, N., van Marle, A. J. et al. 2008, A \& A, 488, L37

Couch, S. M. 2017, RSPTA, 375, 60271

Collins, T. J. B., Frank, A., Bjorkman, J. E. \& Livio, M. 1999, ApJ, 512, 322

Dewey, D., DeLaney, T. and Lazendic, J. S. 2007, RevMexAA, 30, 84 
Eldridge, J. J., Langer, N. \& Tout, C. A. 2011, MNRAS, 414, 3501

Flanagan, K. A., Canizares, C. R., Dewey, D., et al. 2004, ApJ, 605, 230

Foster, A. R., Ji, L., Smith, R. K., \& Brickhouse, N. S. 2012, ApJ, 756, 128

Frank, K., Zhekov, S. A., Park, S. et al. 2016, ApJ, 829, 40

Fruscione, A., McDowell, J. C., Allen, G. E. et al. SPIE Proc. 6270, 62701V, ed. D.R. Silvia \& R.E. Doxsey

Fryer, C. L., Andrews, S., Wesley, E. et al. 2018, ApJ, 856, 6

Gaensler, B. M. \& Wallace, B. J. 2003, ApJ, 594, 326

Gaensler, B. M. \& Slane, P. O. 2006, ARA\&A, 44, 17

Gaetz, T. J., Butt, Y. M., Edgar, R. J., et al. 2000, ApJ, 534, L47

Ghavamian, P., Hughes, J. P., \& Williams, T. B. 2005, ApJ, 635, 365

Ghavamian, P., Raymond, J. C., Blair, W. P., et al. 2009, ApJ, 696, 1307

Ghavamian, P., Long, K. S., Blair, W. P., et al. 2012, ApJ, 750, 39

Ghavamian, P. \& Williams, B. J. 2016, ApJ, 831, 188

Gonzalez, M. \& Safi-Harb, S. 2003, ApJ, 583, L91

Goss, W. M., Shaver, P. A., Zealey, W. J., et al. 1979, MNRAS, 188, 357

Gotthelf, E. V. \& Halpern, J. P. 2009, ApJ, 695, L35

Grefenstette, B. W., Harrison, F. A., Boggs, S. E., et al. 2014, Nature, 506, 339

Gvaramadze, V. V., Langer, N., Fossati, L. et al. 2017, NatAs, 1, 116

Hirai, R., Podsiadlowski, P. \& Yamada, S. 2018, ApJ, 864, 119

Hirschi, R. 2017, in Handbook of Supernovae, ed. A. Alsabti \& P. Murdin. (Cham, Switzerland: Springer International Publishing AG), 1879

Holland-Ashford, T., Lopez, L., Auchettl, K., et al. 2017, ApJ, 844, 84

Houck, J. C. \& Denicola, L. A. 2000, in ASP Conf. Ser. 216, Astronomical Data Analysis Software and Systems IX, ed. N. Manset, C. Veillet, \& D. Crabtree, (San Francisco, CA: ASP), 591

Hughes, J. P., Slane, P. O., Burrows, D. N., et al. 2001, ApJ, 559, L153
Hughes, J. P., Slane, P. O., Park, S., Roming, P. W. A., \& Burrows, D. N. 2003, ApJ, 591, L139

Hwang, U., Holt, S. S. \& Petre, R. 2000, ApJ, 537, L119

Hwang, U., Petre, R. and Flanagan, K. A. 2008, ApJ, 676, 378

Jakobsen, P., Albrecht, R., Barbieri, C. et al. 1991, ApJ, 369, L63

Janka, H.-T., Hanke, F., Hudepohl, L., et al. 2012, PTEP, 2012, aA309J

Janka, H.-T. 2017, ApJ, 837, 84

Kamitsukasa, F., Koyama, K., Tsunemi, H., et al. 2014, PASJ, 66, 648

Katsuda, S., Mori, K., Tsunemi, H., Park, S., Hwang, U., Burrows, D. N., Hughes, J. P. and Slane, P. O. 2008, ApJ, 678, 297

Katsuda, S., Morii, M., Janka, H.-T., et al. 2018a, ApJ, 856, 18

Katsuda, S., Takiwaki, T., Tominaga, N. et al. 2018b, ApJ, 863, 127

Kurfurst, P., Feldmeier, A. \& Krticka, J. 2018, A\&A, 613, 75

Lai, D. 2001, LNP, 578, 424

Langer, N. 2012, ARA\&A, 50, 107

Lazendic, J. S., Dewey, D., Schulz, N. S., \& Canizares, C. R. 2006, ApJ, 651, 250

Leonard, D., Filipenko, A. V., Ganeshalingam, M., et al. 2006, Nature, 440, 505

Lee, H.-G., Koo, B.-C., Moon, D.-S., et al. 2009, ApJ, 706, 441

Lee, J.-J., Park, S., Hughes, J. P., et al. 2010, ApJ, 711, 861

Liu, Z.-W., Tauris, T. M., Roepke, F. K., et al. 2018, IAU Symp., 346, arXiv:1811.08556 [astro-ph.SR]

Lopez, L. A., Ramirez-Ruiz, E., Pooley, D. A. \& Jeltema, T. E. 2009, ApJ, 691, 875

Lopez, L. A., Ramirez-Ruiz, E., Huppenkothen, D., et al. 2011, ApJ, 732, 18

Lopez, L. A. \& Fesen, R. A. 2018, SSRv, 214, 44

Luna, G. J. M., Smith, M. J. S, Dubner, G., Giacani, E. and Casteletti, G. 2016, A\&A, 590, A70

Maoz, D. \& Graur, O. 2017, ApJ, 848, 25

Mitsuda, K., Bautz, M., Inoue, H. et al. 2007, PASJ, 59, S1

Morris, T. \& Podsiadlowski, Ph. 2009, MNRAS, 399,515

Nomoto, K., Tominaga, N., Umeda, H. et al. 2006, NuPhA, 777, 424 
Nordhaus, J., Brandt, T. D., Burrows, A., et al. 2010, PhRvD, 82, 103016

Nordhaus, J., Brandt, T. D., Burrows, A., et al. 2012, MNRAS, 423, 1805

Park, S., Roming, P. W. A., Hughes, J. P. et al. 2002, ApJ, 564, L39

Park, S., Hughes, J. P., Slane, P. O., et al. 2004, ApJ, 602, L33

Park, S., Hughes, J. P., Slane, P. O., et al. 2007, ApJ, 670, L121

Pavlov, G. G., Zavlin, V. E., Aschenbach, B. et al. 2000, ApJ, 531, L53

Rodgers, A. W., Campbell, C. T. \& Whiteoak, J. B. 1960, MNRAS, 121, 103

Scheck, L., Kifondis, K., Janka, H.Th. \& Muller, E. 2006, A\&A, 457, 963

Schenck, A., Park, S., Burrows, D. N. et al. 2014, ApJ, 791, 50

Schenck, A., Park, S. \& Post, S. 2016, AJ, 151, 161

Smith, R. K., Brickhouse, N. S., Liedahl, D. A. \& Raymond, J. C. 2001, ApJ, 556, L91

Smith, N., Arnett, W. D., Bally, J. et al. 2013, MNRAS, 429, 1324

Sukhbold, R., Ertl, T., Woosley, S. E. et al. 2016, ApJ, 821, 38

Thielemann, F. -K., Frohlich, C., Hirschi, R., et al. 2007, PrPNP, 59, 74

Tuohy, I. R., Clark, D. H. \& Burton, W. M. 1982, ApJ, 260, L65

Vink, J., Kaastra, J. S. and Bleeker, J. A. M. 1996, A\&A, 307, L41
Vink, J. 2008, AdSpR, 41, 503

Vink, J. 2012, A\&ARv, 20, 49

Vink J. 2017, in Handbook of Supernovae, ed. A. Alsabti \& P. Murdin. (Cham, Switzerland: Springer International Publishing AG), 2063

Wang, L., Howell, A., Hoflich, P. et al. 2001, ApJ, 550, 1030

Wang, L., Baade, D., Hoflich, P. \& Wheeler, J. C. 2003, 592, 457

Wang, L. \& Wheeler, J. C. 2008, ARA\&A, 46, 433

Weisskopf, M. C., Aldcroft, T. L., Bautz, M. et al. 2005, CIBu, 162, 5

Winkler, P. F. \& Long, K. S. 2006, AJ, 132, 360

Winkler, P. F., Twelker, K., Reith, C. N., \& Long, K. S. 2009, ApJ, 692, 1489

Wongwathanarat, A., Janka, H.-Th. \& Muller, E. 2013, A\&A, 552, 126

Wongwathanarat, A., Janka, H.-T., Muller, E., Pllumbi, E. \& Wanajo, S. 2017, ApJ, 842, 13

Woosley, S. E. \& Weaver, T. A. 1995, ApJS, 101, 181

Woosley, S. E., Heger, A. \& Weaver, T. A. 2002, RvMP, 74, 1015

Yamaguchi, H., Badenes, C., Petre, R., et al. 2014, ApJ, 785, L27

Yang, X.-J., Liu, X.-Q., Li, S.-Y., \& Lu, F.-J. 2014, RAA, 14, 1279 
Table 1. Chandra Observations of G292

\begin{tabular}{lccc}
\hline \hline ObsID & Date & $\begin{array}{c}\text { Exposure } \\
(\mathrm{ks})\end{array}$ & $\begin{array}{c}\text { Roll Angle }^{\mathrm{a}} \\
\left({ }^{\circ}\right)\end{array}$ \\
\hline $6680^{\mathrm{b}}$ & 2006 Sep 13 & 39 & 180 \\
6678 & 2006 Oct 2 & 44 & 157 \\
6679 & 2006 Oct 3 & 154 & 157 \\
8447 & 2006 Oct 7 & 48 & 157 \\
6677 & 2006 Oct 16 & 159 & 140 \\
8221 & 2006 Oct 20 & 65 & 140 \\
\hline
\end{tabular}

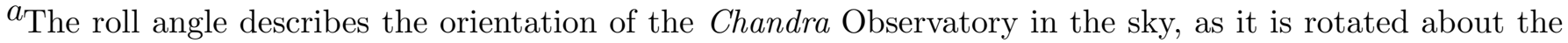
viewing axis to optimally align its solar panels with the Sun.

${ }^{b}$ ObsID 6680 included two ACIS-S chips in addition to four ACIS-I chips. This observation was affected by telemetry saturation which reduced the exposure time by $\sim 25 \%$. The 5 subsequent observations used the four ACIS-I chips only, and were not affected by telemetry saturation. 
Table 2. Average CSM Abundances

\begin{tabular}{|c|c|c|c|c|c|c|c|c|c|}
\hline$N_{\mathrm{H}}$ & $k T$ & $n_{e} t$ & $E M$ & $\mathrm{O}$ & $\mathrm{Ne}$ & $\mathrm{Mg}$ & $\mathrm{Si}$ & S & $\mathrm{Fe}$ \\
\hline$\left(10^{22} \mathrm{~cm}^{-2}\right)$ & $(\mathrm{keV})$ & $\left(10^{12} \mathrm{~cm}^{-3} \mathrm{~s}\right)$ & $\left(10^{56} \mathrm{~cm}^{-3}\right)$ & & & & & & \\
\hline $0.43_{-0.02}^{+0.02}$ & $0.82_{-0.03}^{+0.14}$ & $0.24_{-0.03}^{+0.05}$ & $1.37_{-0.16}^{+0.13}$ & $0.33_{-0.03}^{+0.06}$ & $0.48_{-0.03}^{+0.05}$ & $0.24_{-0.02}^{+0.03}$ & $0.21_{-0.02}^{+0.03}$ & $0.34_{-0.08}^{+0.08}$ & $0.13_{-0.02}^{+0.03}$ \\
\hline
\end{tabular}

Table 3. Line and Continuum Energy Ranges for the EWI Images

\begin{tabular}{lccc}
\hline \hline \multicolumn{1}{c}{ Element/Ion } & Line & Low $^{\mathrm{a}}$ & High $^{\mathrm{a}}$ \\
& $(\mathrm{eV})$ & $(\mathrm{eV})$ & $(\mathrm{eV})$ \\
\hline O He $\alpha$ \& Ly $\alpha$ (O VII \& O VIII) & $510-740$ & $300-510$ & $740-870$ \\
Ne He $\alpha$ (Ne IX) & $890-970$ & $740-870$ & $1120-1160$ \\
Ne Ly $\alpha$ (Ne X) & $1000-1100$ & $740-870$ & $1120-1160$ \\
Mg He $\alpha$ (Mg XI) & $1290-1420$ & $1250-1290$ & $1620-1700$ \\
Mg Ly $\alpha$ (Mg XII) & $1430-1510$ & $1390-1430$ & $1520-1630$ \\
Si He $\alpha$ (Si XIII) & $1750-1930$ & $1620-1700$ & $2020-2120$ \\
S He $\alpha$ (S XV) & $2300-2600$ & $2000-2100$ & $2610-2750$ \\
Fe He $\alpha($ Fe XXV) & $6300-6850$ & $5500-5900$ & $6950-7250$ \\
\hline
\end{tabular}

${ }^{a}$ Low and high energy ranges used to estimate the underlying continuum. 
Table 4. Estimated Shocked Ejecta Masses

\begin{tabular}{|c|c|c|c|c|c|c|c|}
\hline Element & $\begin{array}{c}\text { Entire SNR } \\
\qquad\left(\mathrm{M}_{\odot}\right)\end{array}$ & $\begin{array}{c}\mathrm{NW} \\
\left(\mathrm{M}_{\odot}\right)\end{array}$ & $\begin{array}{c}\mathrm{NE} \\
\left(\mathrm{M}_{\odot}\right)\end{array}$ & $\begin{array}{c}\mathrm{SE} \\
\left(\mathrm{M}_{\odot}\right)\end{array}$ & $\begin{array}{c}\mathrm{SW} \\
\left(\mathrm{M}_{\odot}\right)\end{array}$ & $\begin{array}{c}\text { NW Hem. } \\
\qquad\left(\mathrm{M}_{\odot}\right)\end{array}$ & $\begin{array}{c}\text { SE Hem. } \\
\qquad\left(\mathrm{M}_{\odot}\right)\end{array}$ \\
\hline $\mathrm{O}$ & $0.47_{-0.12}^{+0.23}$ & $0.13_{-0.03}^{+0.06}$ & $0.09_{-0.02}^{+0.03}$ & $0.08_{-0.03}^{+0.05}$ & $0.08_{-0.03}^{+0.05}$ & $0.20_{-0.05}^{+0.09}$ & $0.17_{-0.05}^{+0.10}$ \\
\hline $\mathrm{Ne}$ & $0.57_{-0.10}^{+0.18}$ & $0.16_{-0.03}^{+0.04}$ & $0.10_{-0.01}^{+0.02}$ & $0.08_{-0.02}^{+0.03}$ & $0.11_{-0.03}^{+0.05}$ & $0.27_{-0.04}^{+0.07}$ & $0.19_{-0.04}^{+0.08}$ \\
\hline $\mathrm{Mg}$ & $0.13_{-0.03}^{+0.04}$ & $0.06_{-0.01}^{+0.02}$ & $0.04_{-0.01}^{+0.01}$ & $0.03_{-0.01}^{+0.01}$ & $0.04_{-0.01}^{+0.02}$ & $0.10_{-0.02}^{+0.02}$ & $0.07_{-0.02}^{+0.03}$ \\
\hline $\mathrm{Si}$ & $0.06_{-0.01}^{+0.01}$ & $0.01_{-0.002}^{+0.003}$ & $0.008_{-0.001}^{+0.002}$ & $0.001_{-0.0001}^{+0.0001}$ & $0.005_{-0.001}^{+0.002}$ & $0.02_{-0.004}^{+0.005}$ & $0.002_{-0.0006}^{+0.0006}$ \\
\hline S & $0.02_{-0.01}^{+0.01}$ & $0.003_{-0.001}^{+0.001}$ & $0.002_{-0.0003}^{+0.0003}$ & $0.0^{\dagger}$ & $0.0003_{-0.0001}^{+0.0001}$ & $0.005_{-0.001}^{+0.001}$ & $0.0^{\dagger}$ \\
\hline $\mathrm{Fe}$ & $0.03_{-0.01}^{+0.01}$ & $0.008_{-0.001}^{+0.003}$ & $0.005_{-0.001}^{+0.001}$ & $0.003_{-0.001}^{+0.002}$ & $0.005_{-0.002}^{+0.003}$ & $0.01_{-0.002}^{+0.004}$ & $0.008_{-0.002}^{+0.004}$ \\
\hline Total & $\begin{array}{l}1.29_{-0.28}^{+0.49} \\
\end{array}$ & $\begin{array}{l}0.37_{-0.07}^{+0.13} \\
n^{-0.01}\end{array}$ & $\begin{array}{l}0.24_{-0.04}^{+0.06} \\
x^{-0.00}\end{array}$ & $\begin{array}{l}0.19_{-0.06}^{+0.0010} \\
\text { +0.0. }\end{array}$ & $\begin{array}{l}0.25_{-0.06}^{+0.012} \\
\text { (1) }\end{array}$ & $0.61_{-0.11}^{+0.20}$ & $\begin{array}{l}0.44_{-0.12}^{+0.21} \\
\end{array}$ \\
\hline
\end{tabular}

Note-Ejecta masses are in terms of $f^{1 / 2} d_{6}^{3 / 2} M_{\odot}$, where $d_{6}$ is the distance to the SNR in units of 6 kpc. The mass for the entire SNR is the sum of the six elemental masses calculated for all $\sim 1400$ ejecta regions. The masses for the NW, NE, SE and SW quadrants and the NW and SE hemispheres are for regions where the given element's abundance was higher than the CSM abundance by at least a $3 \sigma$ level of confidence. We note that there may be additional amounts of radiatively cooled ejecta that are emitting predominantly at optical and infrared wavelengths. For example, significant ejecta emission from [O III], [N II], and [N IV] has been detected in a crescent-shaped structure in the remnant's southeast known as the "spur" (e.g., Ghavamian et al. 2005, 2009, 2012; Winkler \& Long 2006; Winkler et al. 2009). Thus, the total ejecta mass in G292 may be higher than our estimates here, that only consider the X-ray-emitting ejecta.

*Hem.: Hemisphere

$\dagger$ We note that, in the optical band, a few S-rich ejecta features have been detected in the southeastern parts of the remnant (Winkler \& Long 2006). This suggests that there may be dense clumps of S-rich ejecta in the southeast that have radiatively cooled to produce optical emission but not X-rays.

Table 5. Estimated Shocked CSM Masses

\begin{tabular}{lc}
\hline \hline Component & Mass $\left(\mathrm{M}_{\odot}\right)$ \\
\hline Equatorial ring & $1.7_{-0.1}^{+0.1}$ \\
Outer spherical CSM & $5.0_{-0.6}^{+0.6}$ \\
Centrally projected spherical CSM & $6.8_{-0.7}^{+1.0}$ \\
Total & $13.5_{-1.4}^{+1.7}$ \\
\hline \multicolumn{2}{c}{ NoTE-The masses are in terms of $f^{1 / 2} d_{6}^{3 / 2} M_{\odot}}$, \\
where $d_{6}$ is the distance to the SNR in units of \\
6 kpc. The "spherical CSM" includes all the re- \\
gions with spectrally soft, diffuse emission. This \\
includes the "outer spherical CSM" regions which \\
are projected close to the outer boundary of the \\
remnant, and the "centrally projected spherical \\
CSM" regions which are projected towards the \\
central parts of the remnant.
\end{tabular}


Table 6. Average Ejecta Abundances

\begin{tabular}{lcccccc}
\hline \hline \multicolumn{1}{c}{ Region } & $\mathrm{O}$ & $\mathrm{Ne}$ & $\mathrm{Mg}$ & $\mathrm{Si}$ & $\mathrm{S}$ & $\mathrm{Fe}$ \\
\hline Entire SNR: & $1.63_{-0.02}^{+0.05}$ & $2.63_{-0.01}^{+0.03}$ & $1.24_{-0.01}^{+0.01}$ & $0.84_{-0.01}^{+0.01}$ & $1.55_{-0.04}^{+0.06}$ & $1.26_{-0.02}^{+0.07}$ \\
NW quadrant: & $1.82_{-0.03}^{+0.09}$ & $3.18_{-0.02}^{+0.06}$ & $1.47_{-0.01}^{+0.03}$ & $0.89_{-0.01}^{+0.02}$ & $1.74_{-0.08}^{+0.00}$ & $1.51_{-0.03}^{+0.15}$ \\
NE quadrant: & $1.43_{-0.02}^{+0.05}$ & $2.44_{-0.02}^{+0.04}$ & $1.12_{-0.01}^{+0.02}$ & $0.96_{-0.02}^{+0.03}$ & $1.64_{-0.08}^{+0.00}$ & $1.54_{-0.02}^{+0.08}$ \\
SE quadrant: & $1.75_{-0.05}^{+0.17}$ & $2.38_{-0.04}^{+0.04}$ & $1.20_{-0.02}^{+0.05}$ & $0.65_{-0.09}^{+0.11}$ & $1.35_{-0.74}^{+1.08}$ & $0.83_{-0.06}^{+0.21}$ \\
SW quadrant: & $1.51_{-0.04}^{+0.09}$ & $2.22_{-0.02}^{+0.05}$ & $1.08_{-0.01}^{+0.02}$ & $0.68_{-0.01}^{+0.02}$ & $1.16_{-0.07}^{+0.08}$ & $0.78_{-0.06}^{+0.09}$ \\
NW Hemisphere: & $1.68_{-0.02}^{+0.06}$ & $2.76_{-0.01}^{+0.04}$ & $1.29_{-0.01}^{+0.02}$ & $0.87_{-0.01}^{+0.02}$ & $1.63_{-0.06}^{+0.06}$ & $1.63_{-0.02}^{+0.11}$ \\
SE Hemisphere: & $1.56_{-0.03}^{+0.08}$ & $2.42_{-0.02}^{+0.06}$ & $1.16_{-0.01}^{+0.02}$ & $0.65_{-0.03}^{+0.03}$ & $0.92_{-0.12}^{+0.13}$ & $0.82_{-0.03}^{+0.07}$ \\
\hline
\end{tabular}

Note-Abundances are with respect to solar (Anders \& Grevesse 1989). Uncertainties are averages of the $90 \%$ confidence level uncertainties. The locations of the quadrants and hemispheres are shown in Fig. 11.

Table 7. Estimated Shocked Ejecta Masses - Shell Regions

\begin{tabular}{lcccc}
\hline \hline \multirow{2}{*}{ Element } & $\mathrm{NW}$ & $\mathrm{NE}$ & $\mathrm{SE}$ & $\mathrm{SW}$ \\
& $\left(\mathrm{M}_{\odot}\right)$ & $\left(\mathrm{M}_{\odot}\right)$ & $\left(\mathrm{M}_{\odot}\right)$ & $\left(\mathrm{M}_{\odot}\right)$ \\
\hline $\mathrm{O}$ & $0.10_{-0.03}^{+0.05}$ & $0.06_{-0.01}^{+0.02}$ & $0.04_{-0.01}^{+0.02}$ & $0.02_{-0.01}^{+0.01}$ \\
$\mathrm{Ne}$ & $0.13_{-0.02}^{+0.03}$ & $0.07_{-0.01}^{+0.02}$ & $0.05_{-0.01}^{+0.02}$ & $0.05_{-0.01}^{+0.01}$ \\
$\mathrm{Mg}$ & $0.05_{-0.01}^{+0.01}$ & $0.02_{-0.003}^{+0.005}$ & $0.02_{-0.004}^{+0.006}$ & $0.02_{-0.003}^{+0.004}$ \\
$\mathrm{Si}$ & $0.006_{-0.002}^{+0.001}$ & $0.006_{-0.001}^{+0.001}$ & $0.0004_{-0.0001}^{+0.0001}$ & $0.005_{-0.001}^{+0.001}$ \\
$\mathrm{~S}$ & $0.002_{-0.001}^{+0.001}$ & $0.001_{-0.0002}^{+0.0002}$ & 0.0 & $0.0003_{-0.0001}^{+0.0001}$ \\
$\mathrm{Fe}$ & $0.005_{-0.002}^{+0.001}$ & $0.003_{-0.001}^{+0.001}$ & $0.002_{-0.0004}^{+0.0003}$ & $0.002_{-0.001}^{+0.001}$ \\
Total & $0.29_{-0.06}^{+0.10}$ & $0.16_{-0.02}^{+0.04}$ & $0.11_{-0.03}^{+0.05}$ & $0.10_{-0.02}^{+0.03}$ \\
\hline
\end{tabular}

Note-Ejecta masses are in terms of $f^{1 / 2} d_{6}^{3 / 2} M_{\odot}$, where $d_{6}$ is the distance to the SNR in units of $6 \mathrm{kpc}$. The masses are calculated for ejecta regions where the given element's abundance was higher than the CSM abundance by at least a $3 \sigma$ level of confidence. Only regions projected between the estimated positions of the RS and CD (Bhalerao et. al. 2015) are included in this table. These "shell" regions are shown in Fig. 13a. 

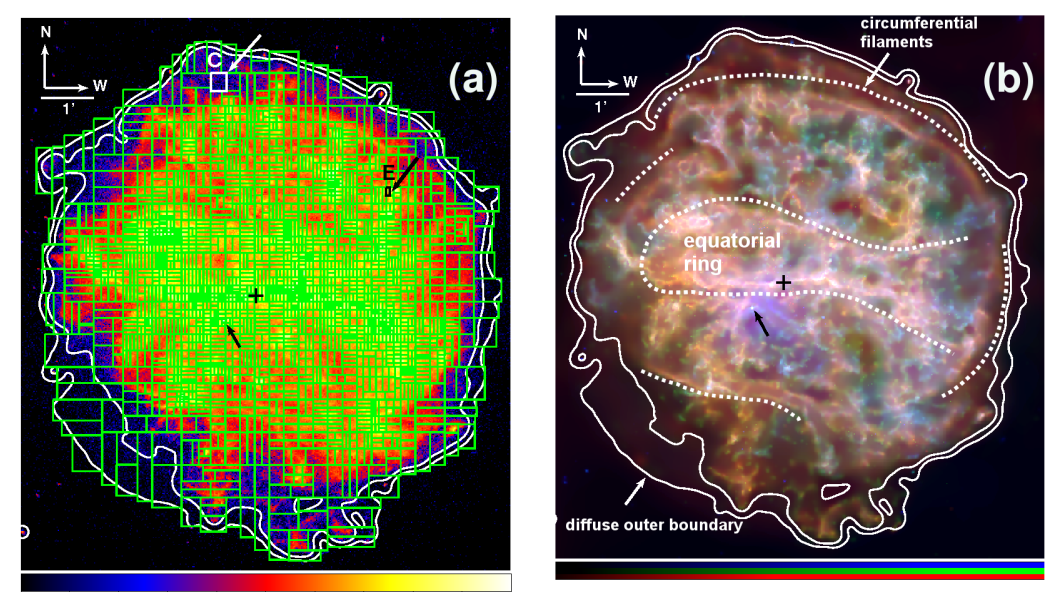

$\begin{array}{llll}8.08 \mathrm{e}-08 & 1.30 \mathrm{e}-07 & 4.59 \mathrm{e}-07 & 1.72 \mathrm{e}-06\end{array}$
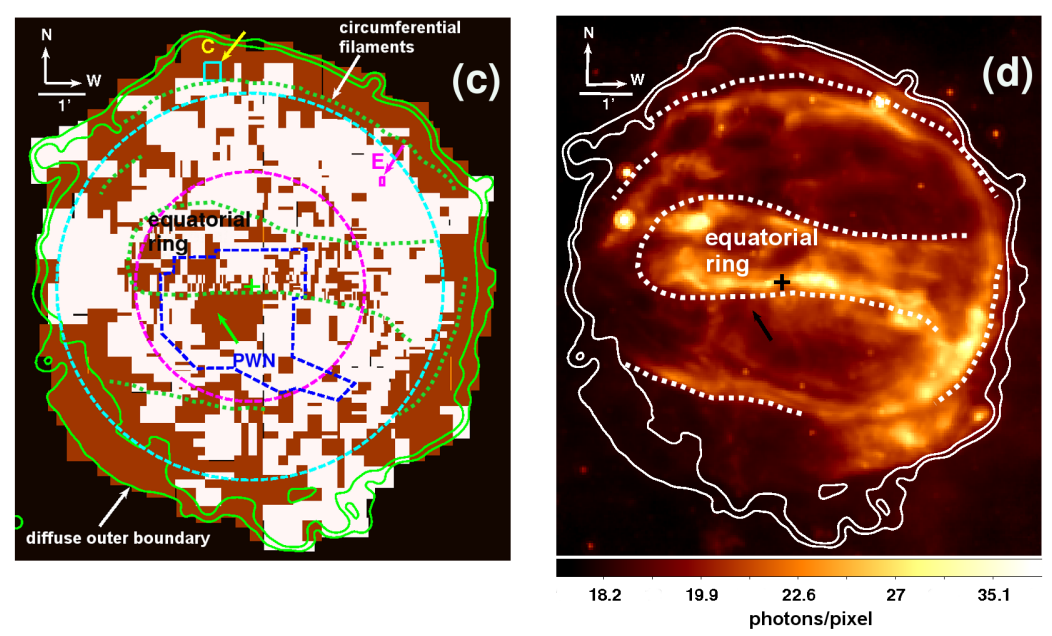

Figure 1. (a) ACIS-I broadband image of G292 (0.3-8 keV) overlaid with the 2147 regions (green boxes) made with our adaptive mesh method. Each region contains 5000 counts. (b) ACIS-I three-color image of G292 (red = 0.3-0.8 keV, green $=0.8-1.7 \mathrm{keV}$ and blue $=1.7-8.0 \mathrm{keV})$. (c) Ejecta and CSM map constructed based on measured abundances for $\mathrm{O}, \mathrm{Ne}, \mathrm{Mg}, \mathrm{Si}, \mathrm{S}$ and $\mathrm{Fe}$. Ejecta-dominated regions are white, while CSM dominated regions are brown. The overlaid dashed circles are our estimated locations of the reverse shock (RS, magenta) and contact discontinuity (CD, cyan), whose positions were inferred from radial velocity measurements of ejecta knots (Bhalerao et. al. 2015). The blue-dashed line marks the location of the 310 PWN regions. (d) $24 \mu \mathrm{m}$ Spitzer image of G292 (Ghavamian et al. 2012). In all four images, the outermost contours (green in $a, c$ and white in $b, d$ ) mark the outer boundary of the SNR in the X-ray band $(\mathrm{E}=0.3-8 \mathrm{keV})$. The cross at the center marks the optical expansion center (Winkler et al. 2009), and the arrow southeast of the center marks the position of the pulsar (PSR J1124-5916). The dashed curves in $b, c$ and $d$ highlight the locations of the thin circumferential filaments and equatorial ring which were previously identified as candidate CSM regions in X-ray (Gonzalez \& Safi-Harb 2003; Park et al. 2002, 2007) and infrared studies (Lee et al. 2009; Ghavamian et al. 2012). The regions labeled "C" and "E" in $a$ and $c$, are sample CSM-dominated and ejecta-dominated regions respectively, whose spectra are shown in Fig. 2. 

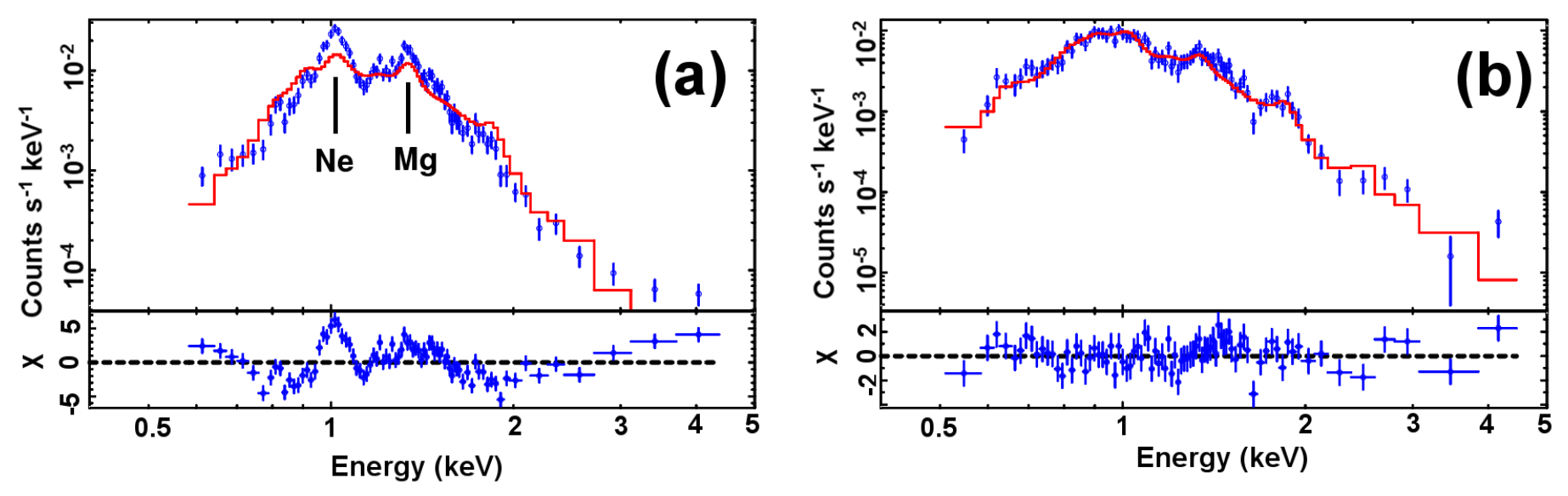

Figure 2. (a) An example spectrum of an ejecta region (region "E" marked in Figs. 1a \& c), showing strong emission lines for $\mathrm{Ne}$ and $\mathrm{Mg}$. The red line is the fitted plane shock model assuming CSM abundances. The large residuals (bottom panel) indicate a poor fit resulting in a high $\chi^{2} / \nu$ value of 6.6 for this region. (b) An example spectrum of a CSM-dominated region near the outer boundary (region "C" marked in Figs. 1a \& c) showing low abundances and thus giving a good fit $\left(\chi^{2} / \nu=1.2\right)$ to the CSM model. 

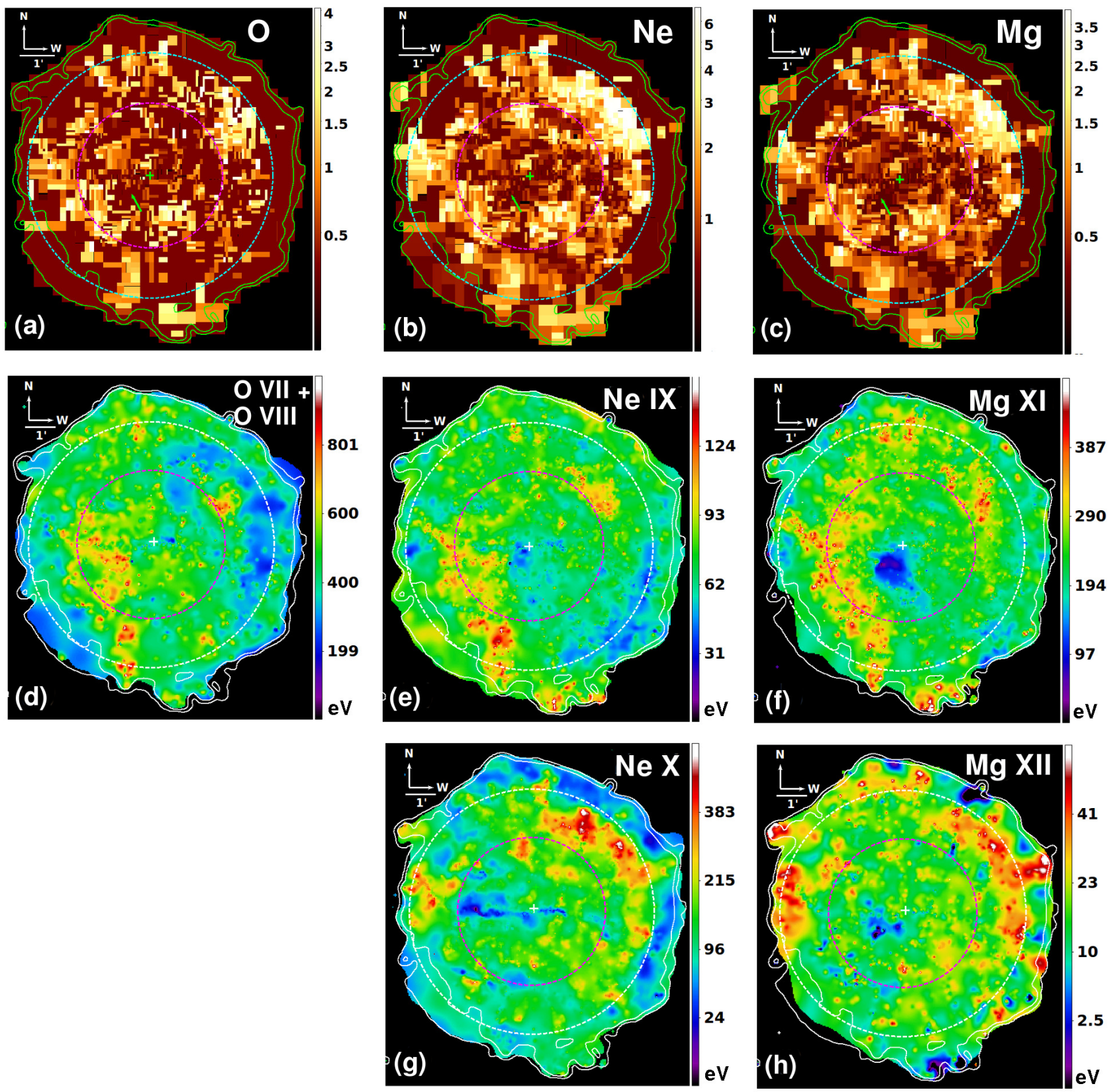

Figure 3. 

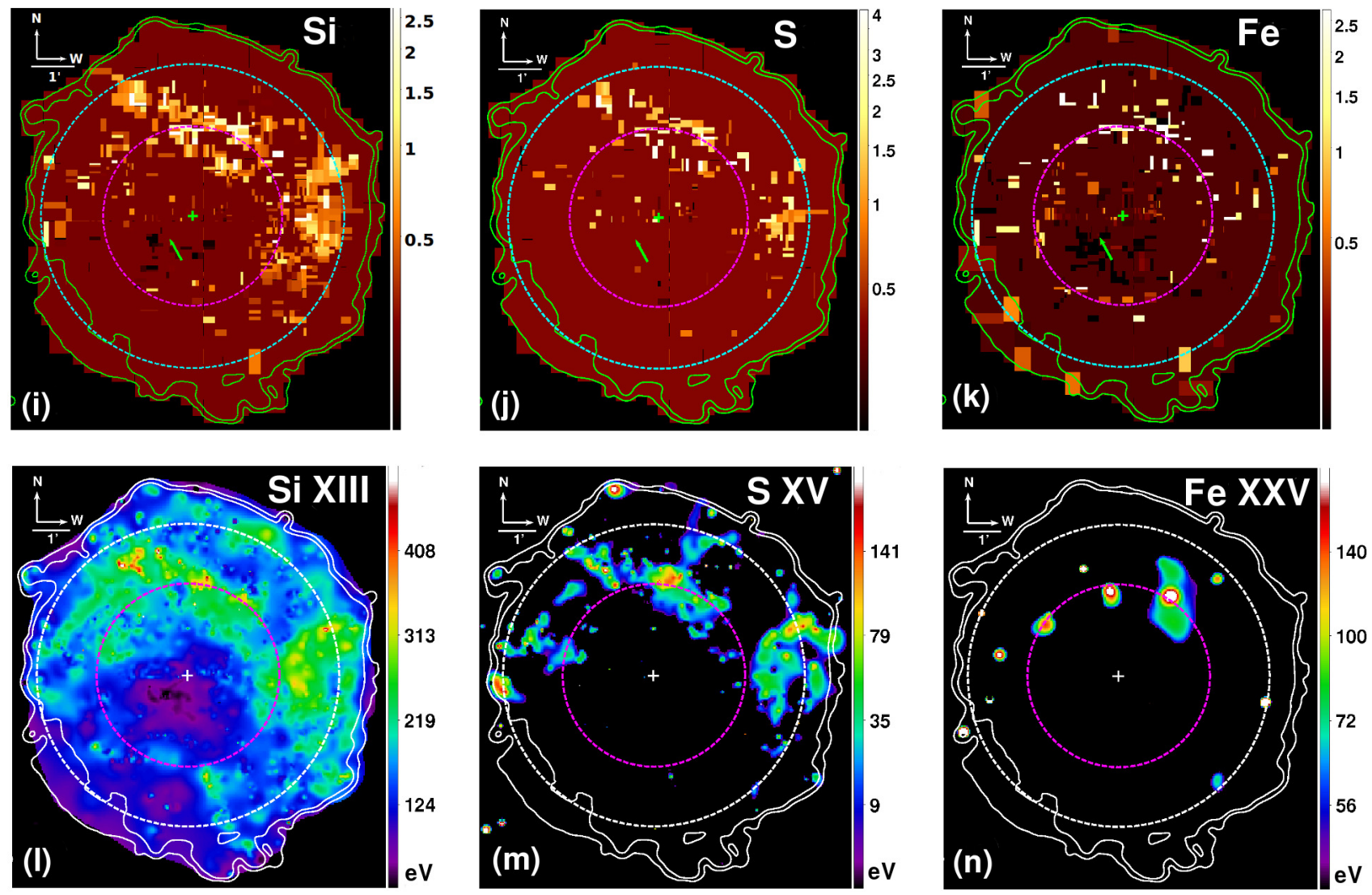

Figure 3. Top panels (a, b, c, i, j \& k): O, Ne, Mg, Si, S and Fe abundances (in units of solar, Anders \& Grevesse 1989). The magenta and cyan dashed-circles represent the estimated locations of the RS and CD respectively (Bhalerao et. al. 2015). The green contours mark the outer boundary of the SNR in the X-rays based on the 0.3-8 keV band ACIS-I image. The cross marks the optical expansion center (Winkler et al. 2009), and the arrow marks the pulsar (PSR J1124-5916). Lower panels (d, e, f, g, h, l, m \& n): O, Ne, Mg, Si, S and Fe line EW maps. 

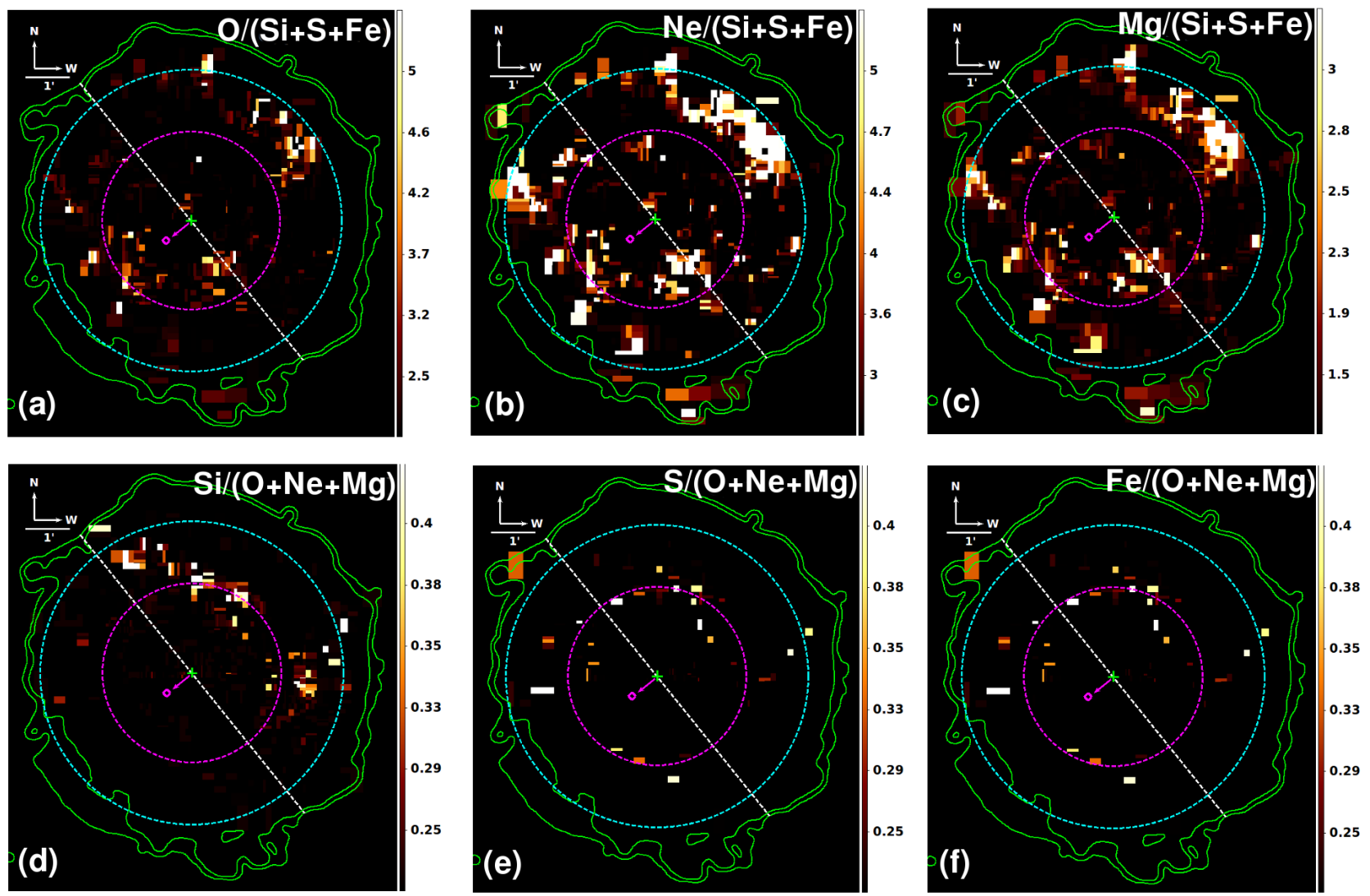

Figure 4. Abundance ratio maps for the ejecta-dominated regions. The green cross marks the optical expansion center (Winkler et al. 2009) and the magenta diamond marks the position of the pulsar. The magenta arrow indicates the suggested direction of the pulsar's kick. The white dashed-line is perpendicular to the direction of the pulsar's kick and divides the remnant into the northwestern and southeastern hemispheres. The dashed magenta circle represents the estimated position of the RS, and the dashed-cyan circle represents the estimated position of the CD (Bhalerao et. al. 2015). Green contours are the outer boundary of the SNR in X-rays based on the 0.3-8 keV band ACIS-I image. 


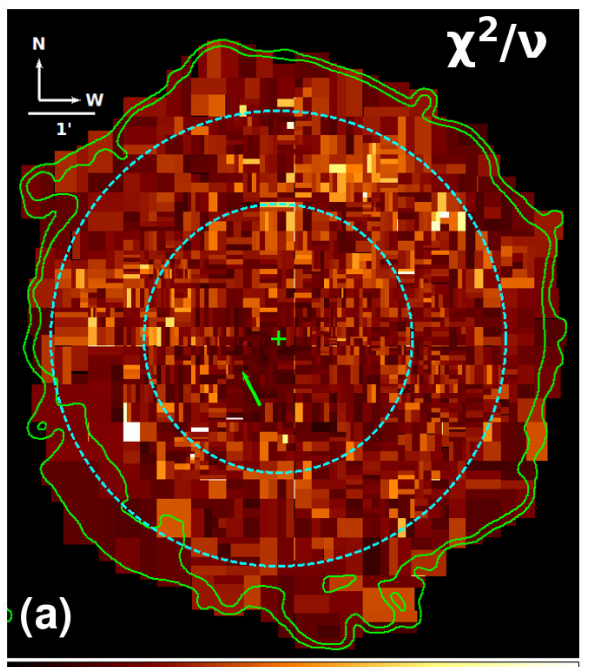

$\begin{array}{llllll}0.88 & 1.4 & 1.7 & 1.9 & 2 & 2.2\end{array}$

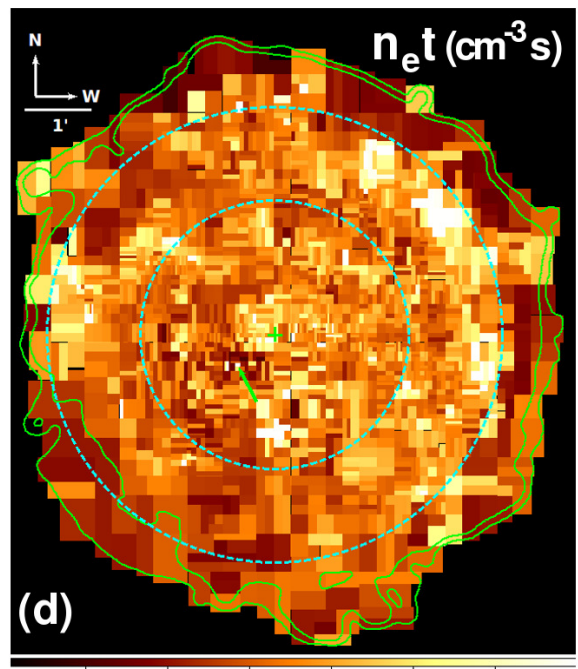

$3.39 e+10 \quad 3.65 e+11 \quad 2.76 e+12$

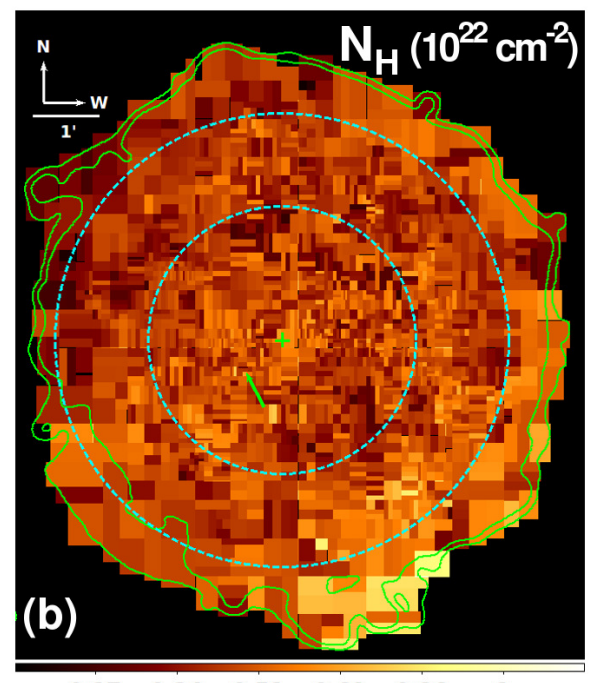

$\begin{array}{llllll}0.17 & 0.34 & 0.51 & 0.69 & 0.86 & 1\end{array}$

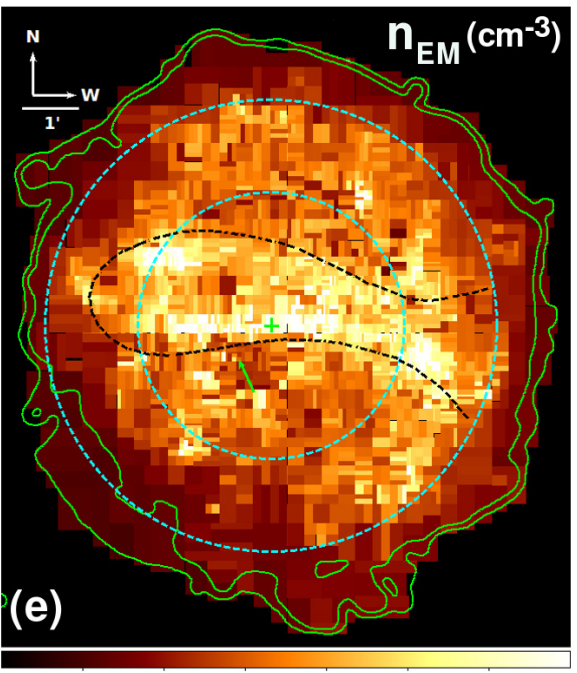

$\begin{array}{llllll}0.84 & 3.3 & 7.5 & 13 & 21 & 30\end{array}$
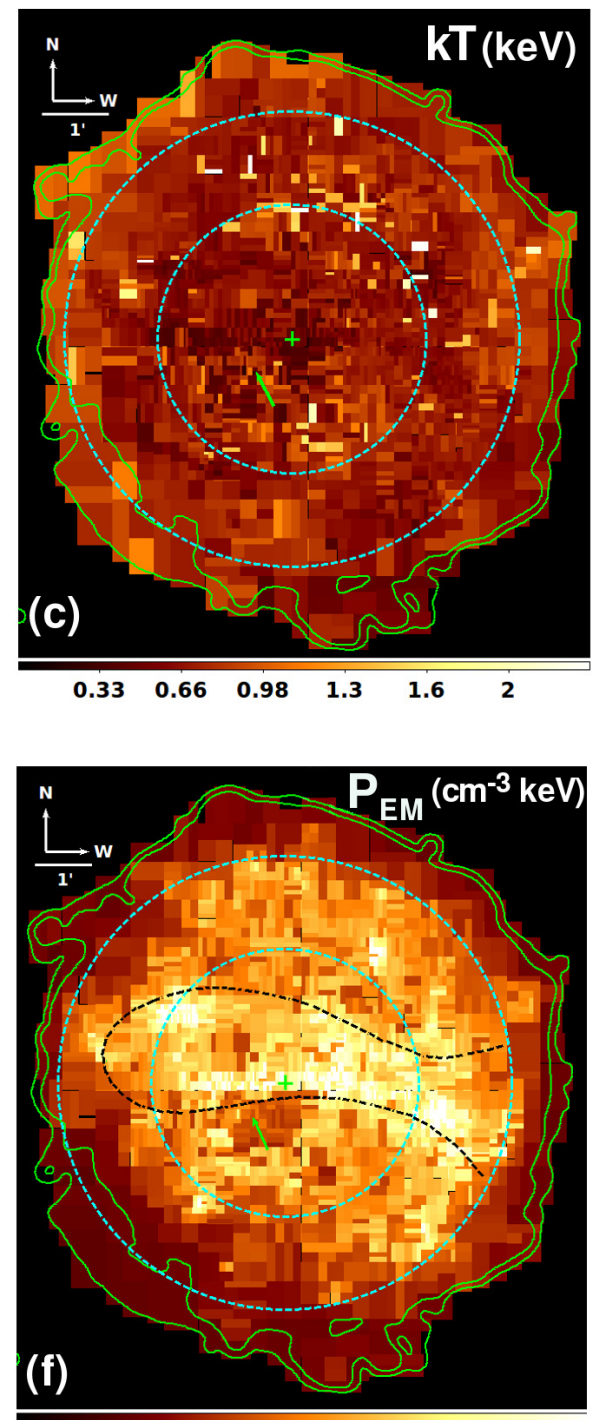

$\begin{array}{llllll}0.49 & 2 & 4.4 & 7.8 & 12 & 18\end{array}$

Figure 5. (a): Reduced $\operatorname{chi}^{2}\left(\chi^{2} / \nu\right)$, (b): foreground column $\left(N_{H}\right),(\mathbf{c})$ : electron temperature $(k T),(\mathbf{d})$ : ionization timescale $\left(n_{e} t\right)$, (e): pseudo electron density $\left(n_{E M}\right)$ and (f): pseudo thermal pressure $\left(P_{E M}\right)$ distribution maps. For all these maps, the green contours mark the outer boundary of the SNR in the X-ray band $(\mathrm{E}=0.3-8 \mathrm{keV})$, the green cross marks the optical expansion center, the green arrow marks the pulsar, the inner and outer cyan circles mark the estimated positions of the RS and CD respectively (Bhalerao et. al. 2015). 

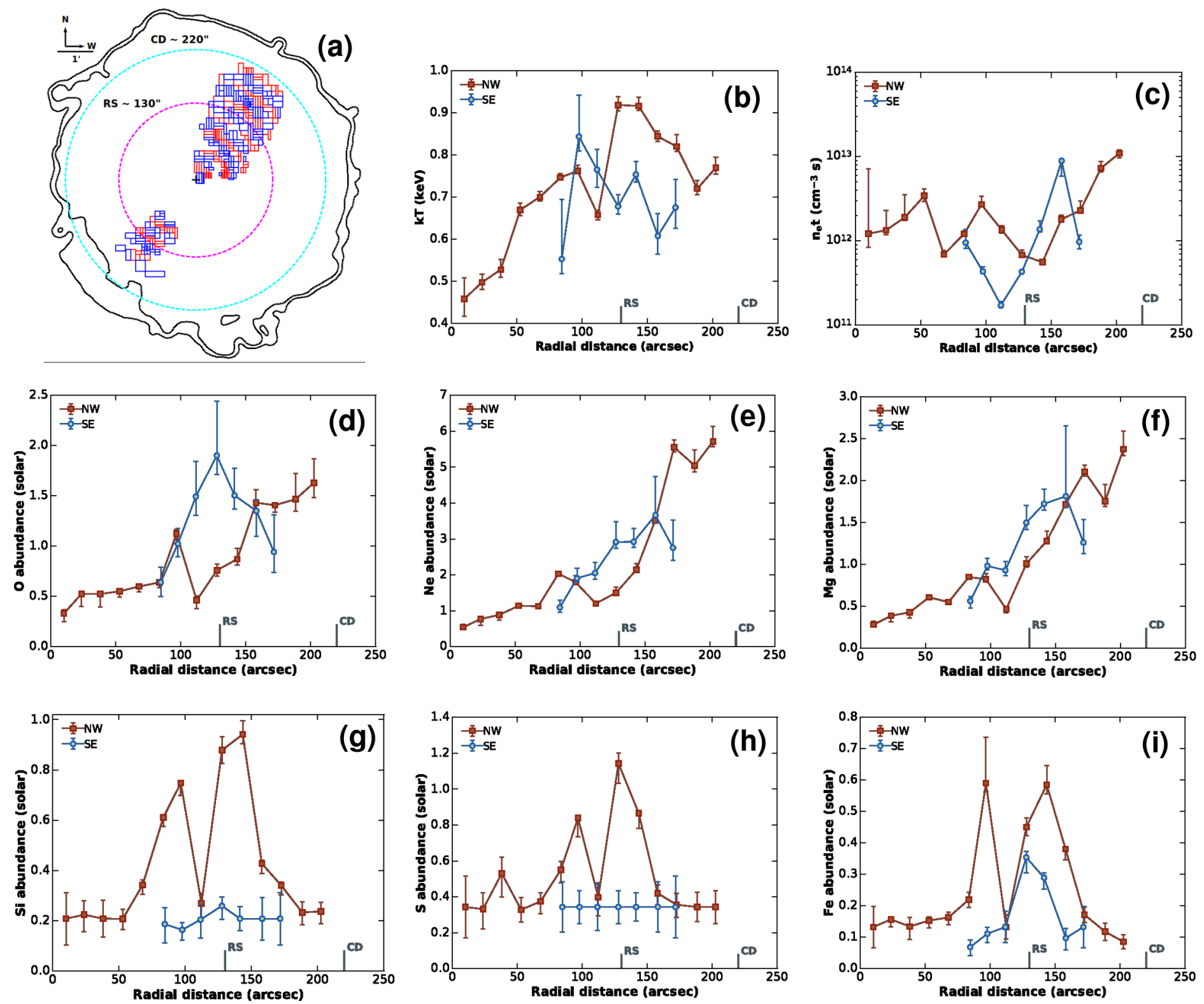

Figure 6. (a) Outline of G292 showing the ejecta regions used for making the radial profiles. The regions were divided into $15^{\prime \prime}$ sections starting at the center and moving outwards radially. These radial slices are shown in alternating blue and red colors. Black contours are the outer boundary of the SNR in X-rays based on the 0.3-8 keV band ACIS-I image. The magenta and cyan dashed-circles represent the estimated locations of the RS and CD respectively based on the overall ejecta's kinematic structure in X-rays (Bhalerao et. al. 2015). Average values from each radial section are plotted against the distance from the expansion center (marked with a black cross). The NW regions are plotted in brown, and the SE regions in blue. The plotted parameters are: (b): $\mathrm{kT}(\mathrm{keV}),(\mathbf{c}): \mathrm{n}_{\mathrm{e}} \mathrm{t}\left(\mathrm{cm}^{-3} \mathrm{~s}\right),(\mathbf{d}): \mathrm{O},(\mathbf{e})$ : $\mathrm{Ne},(\mathbf{f}): \mathrm{Mg},(\mathrm{g})$ : $\mathrm{Si},(\mathbf{h}): \mathrm{S} \&(\mathbf{i})$ : Fe abundance. The short vertical lines on the horizontal axes (labeled "RS" and "CD") represent the estimated locations of the RS and CD respectively. 


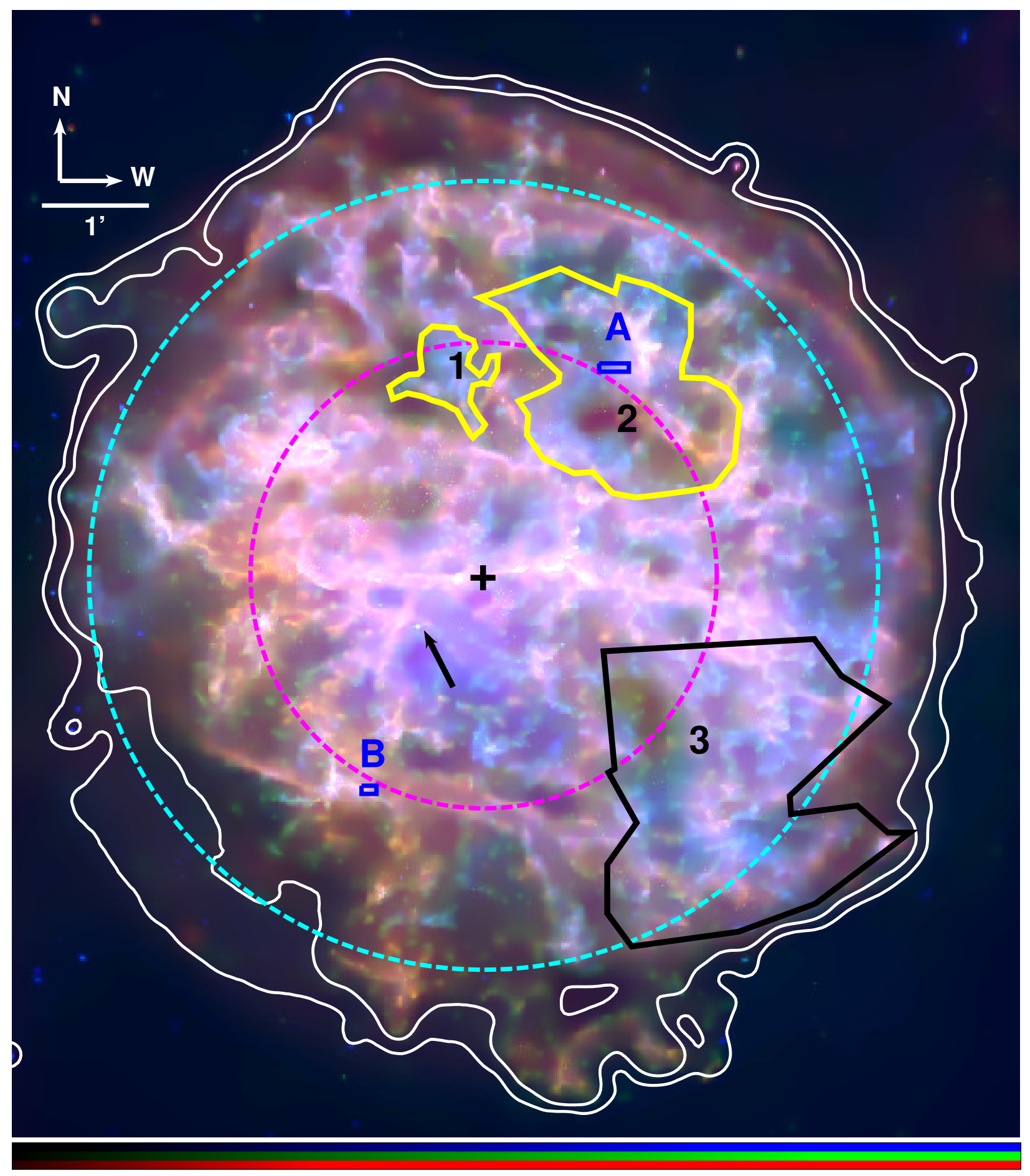

Figure 7. ACIS-I three-color image of G292 showing the extraction regions for detecting Fe emission. Red $=0.3-0.8$ $\mathrm{keV}$, green $=0.8-1.7 \mathrm{keV}$ and blue $=1.7-8.0 \mathrm{keV}$. White contours are the outer boundary of the SNR in X-rays based on the $0.3-8 \mathrm{keV}$ band ACIS-I image. The black cross and arrow mark the positions of the optical expansion center and the pulsar respectively. The dashed magenta circle represents the estimated position of the RS, and the dashed-cyan circle represents the estimated position of the CD (Bhalerao et. al. 2015). 

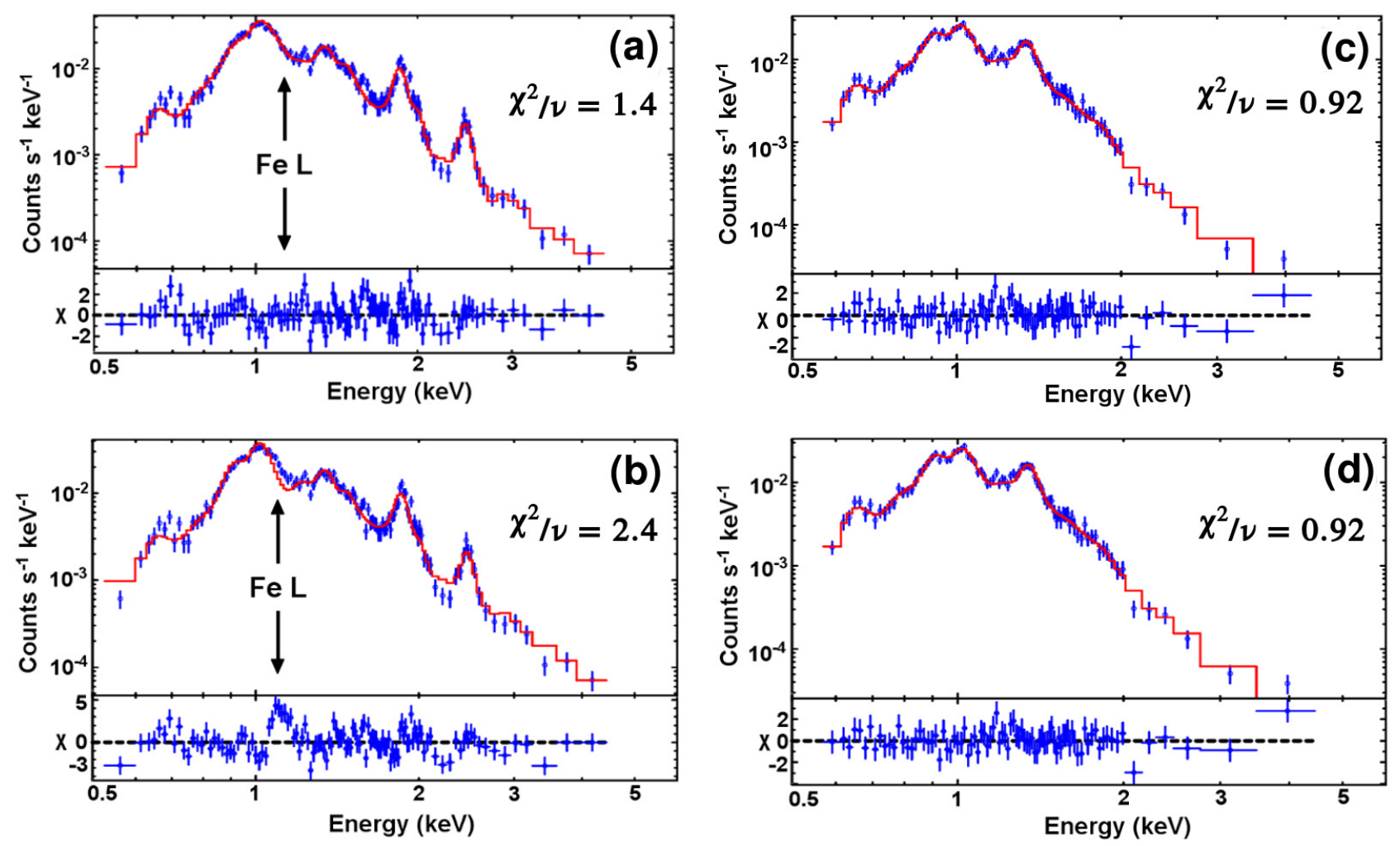

Figure 8. (a \& b): Spectral model fits to the X-ray spectrum of a small Fe-rich region (region A identified in Fig. 7). In (a) the Fe abundance is varied, and the best-fit value is $9.8_{-7.0}^{+15} \times$ solar. In (b), for comparison, we fixed the Fe abundance at the average CSM value $(0.13 \times$ solar $)$, resulting in large residuals at $E \sim 1.2 \mathrm{keV}$ for the Fe L line complex. The abundances of the other elements $(\mathrm{O}, \mathrm{Ne}, \mathrm{Mg}, \mathrm{Si}, \mathrm{S}, \mathrm{Ar}$ and $\mathrm{Ca})$ are free and the abundance of $\mathrm{Ni}$ is tied to that of $\mathrm{Fe}$ in both model fits. The red line is the fitted plane shock model, the blue markers represent data. Fixing Fe increases $\chi^{2} / \nu$ from 1.4 (a) to 2.4 (b). (c \& d): Spectral model fits to the X-ray spectrum of an Fe-poor region (region B in Fig. 7). In (c) we varied the Fe abundance, and the best-fit value is $0.25_{-0.14}^{+0.29} \times$ solar, which is consistent with the average CSM value. In (d), for comparisons, we fixed the Fe abundance at the CSM value ( $0.13 \times$ solar), which is not statistically distinguished from the model fit shown in (c). 

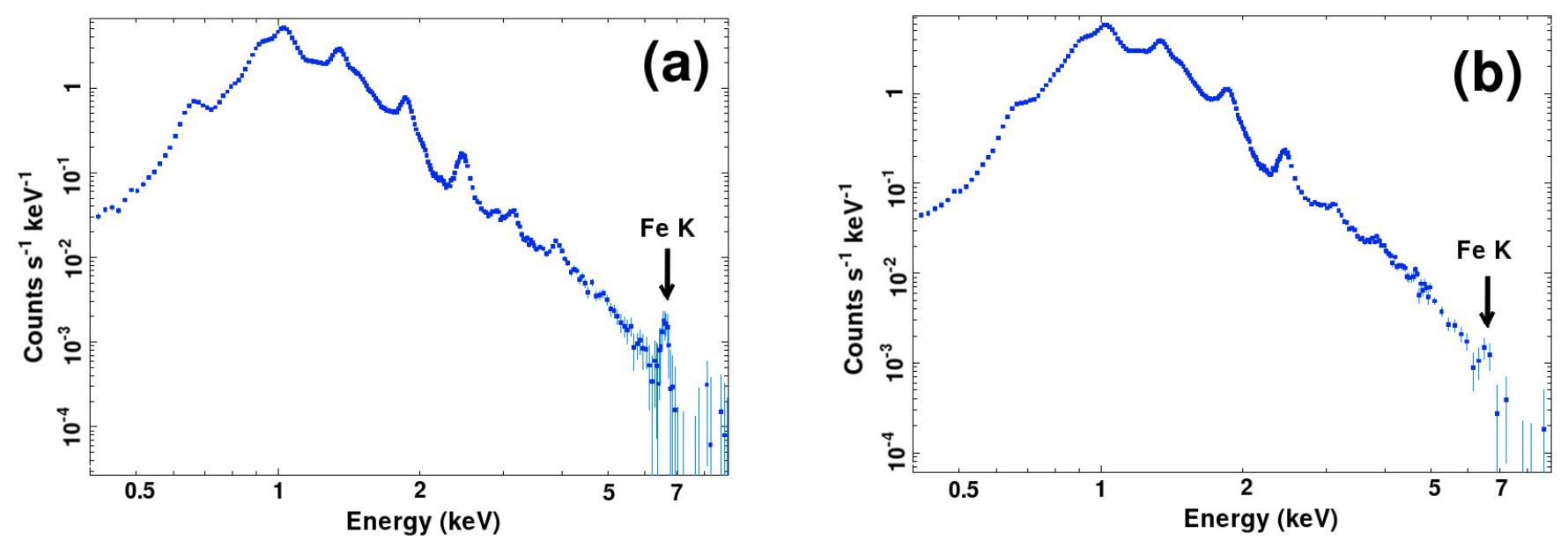

Figure 9. (a): X-ray spectrum extracted from the combined regions labeled 1 and 2 in Fig. 7 showing the Fe K-shell line at $6.6 \mathrm{keV}$. These two regions have $\sim 1.2 \times 10^{6}$ counts in total. (b): X-ray spectrum of an ejecta-dominated region in the southwest (region 3 in Fig. 7) in which the Fe K-shell line detection is only marginal. This regional spectrum has $\sim 1.7 \times 10^{6}$ counts, which are comparable to those in Fig. 9a.

Fe K Line Center Energy

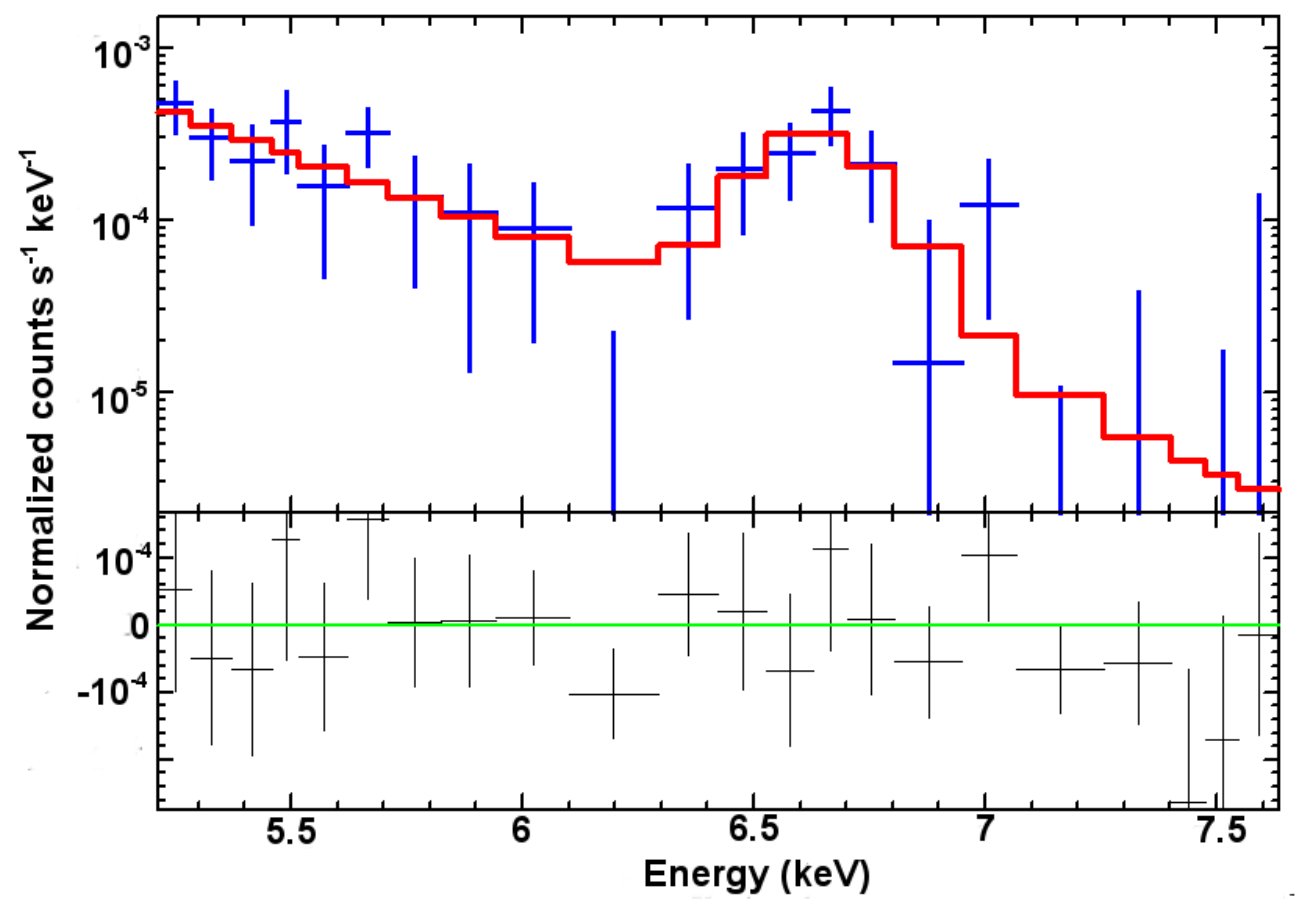

Figure 10. X-ray spectrum of 31 combined Fe-rich northern regions (seen as white boxes in Fig. 3k). The spectrum was binned at 20 counts per bin, and fitted with a Gaussian model. Data bins are in blue, and the model showing the fitted line center energy at $6.62 \mathrm{keV}$ is in red. The bottom panel shows the residuals $\left(\chi^{2} / \nu \sim 1\right)$. 

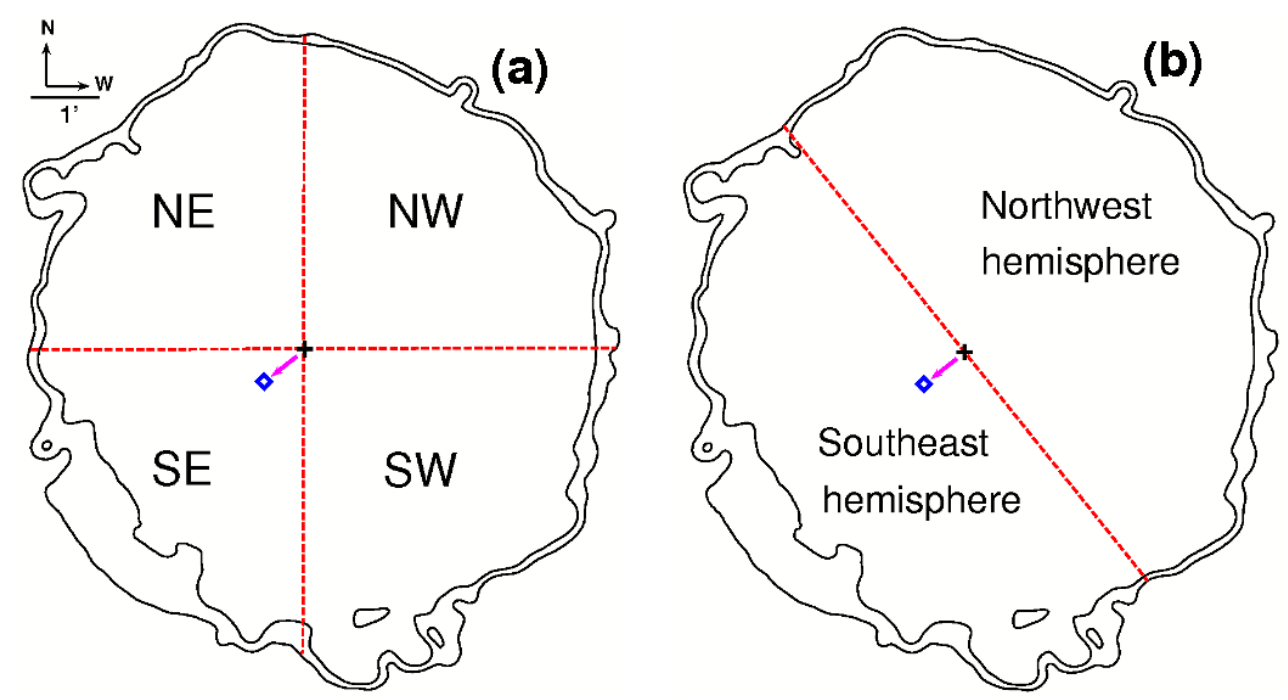

Figure 11. (a): Division of G292 into four quadrants: NW, NE, SE and SW (b): division of G292 into two hemispheres: the northwestern hemisphere, and the southeastern hemisphere to which the pulsar (PSR J1124-5916) has apparently been displaced. Black contours are the outer boundary of the SNR in X-rays based on the 0.3-8 keV band ACIS-I image. The black cross marks the position of the optical expansion center of the SNR (Winkler et al. 2009). The location of pulsar is shown by a blue diamond and the presumed direction of the pulsar's kick is shown by a magenta arrow. The red dashed-line dividing the northwestern and southeastern hemispheres is perpendicular to the direction of the pulsar's presumed kick. 


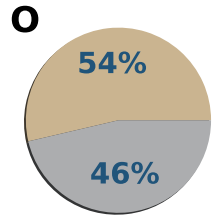

\section{Si}

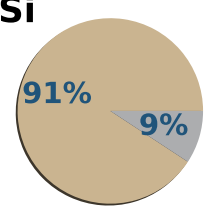

$\mathrm{Ne}$

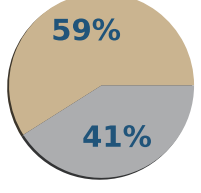

S

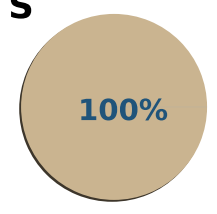

Mg

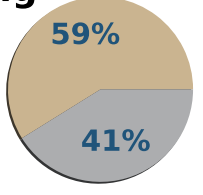

$\mathrm{Fe}$

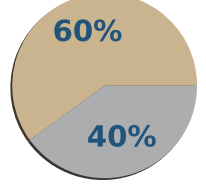

\section{Total}

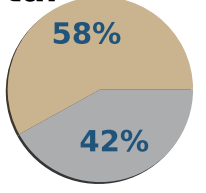

\section{NW Hemisphere \\ SE Hemisphere}

Figure 12. Pie charts showing the fractional distribution of ejecta masses for $\mathrm{O}, \mathrm{Ne}, \mathrm{Mg}, \mathrm{Si}, \mathrm{S}, \mathrm{Fe}$ and the total of these six elements, between the northwestern hemisphere and the southeastern hemisphere (as shown in Fig. 11b). Ejecta regions that had the abundance of a given element greater than the CSM abundance by at least a $3 \sigma$ confidence level were considered for these plots. The northwestern hemisphere dominates the southeast especially for $\mathrm{Si}, \mathrm{S}$, and Fe, where it accounts for $\gtrsim 60 \%$ of the ejecta mass. The uncertainties in the mass estimates are approximately $\pm 15 \%$. We note that there may be additional amounts of radiatively cooled O-, Ne-, and Mg-rich ejecta that are emitting in the optical and infrared bands but not in X-rays. These cooler ejecta have been detected mainly in a crescent-shaped structure known as the "spur" in the southeast (Ghavamian et al. 2005, 2009, 2012; Winkler \& Long 2006; Winkler et al. 2009). Thus, the mass-asymmetry between the NW and SE may be reduced somewhat by contributions from these cooler ejecta, however this may mainly affect the lighter O-group elements rather than the heavier Si, S, and Fe. 
(a)

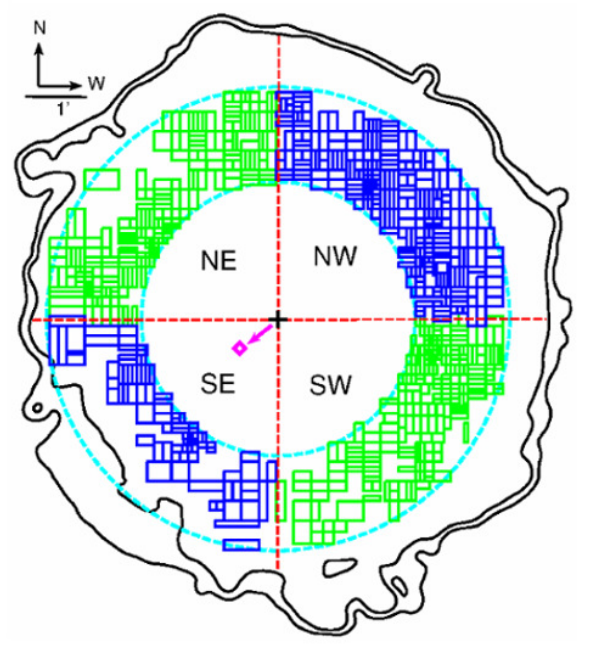

(b) o

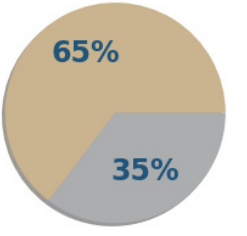

$\mathrm{Ne}$

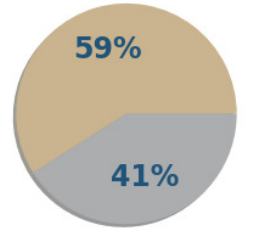

Mg

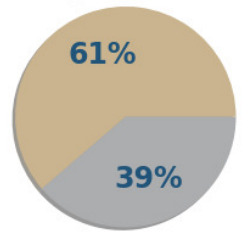

Figure 13. Pie charts showing the fractional distribution of ejecta masses for $\mathrm{O}, \mathrm{Ne}$ and $\mathrm{Mg}$ between the combined NW and SE quadrants (blue regions in (a)), and the combined NE and SW quadrants (green regions in (a)). In order to exclude regions projected towards the central regions of the SNR, only ejecta regions between the estimated locations of the RS and CD (inner and outer dashed-cyan circles respectively) were used in this comparison. The elemental ejecta masses for these "shell" regions are listed in Table 7. Ejecta regions that had the abundance of a given element greater than the CSM abundance by at least a $3 \sigma$ confidence level were considered in these pie charts. The uncertainties in the mass estimates are approximately $\pm 20 \%$. We note that contributions from cooler $\mathrm{O}-$-, Ne-, and Mg-rich ejecta in the southeast, that are emitting in the optical and infrared bands but not in X-rays, would further increase the mass asymmetry between the $\mathrm{NW}+\mathrm{SE}$ (brown sectors in the pie charts) and $\mathrm{NE}+\mathrm{SW}$ directions (gray sectors). 

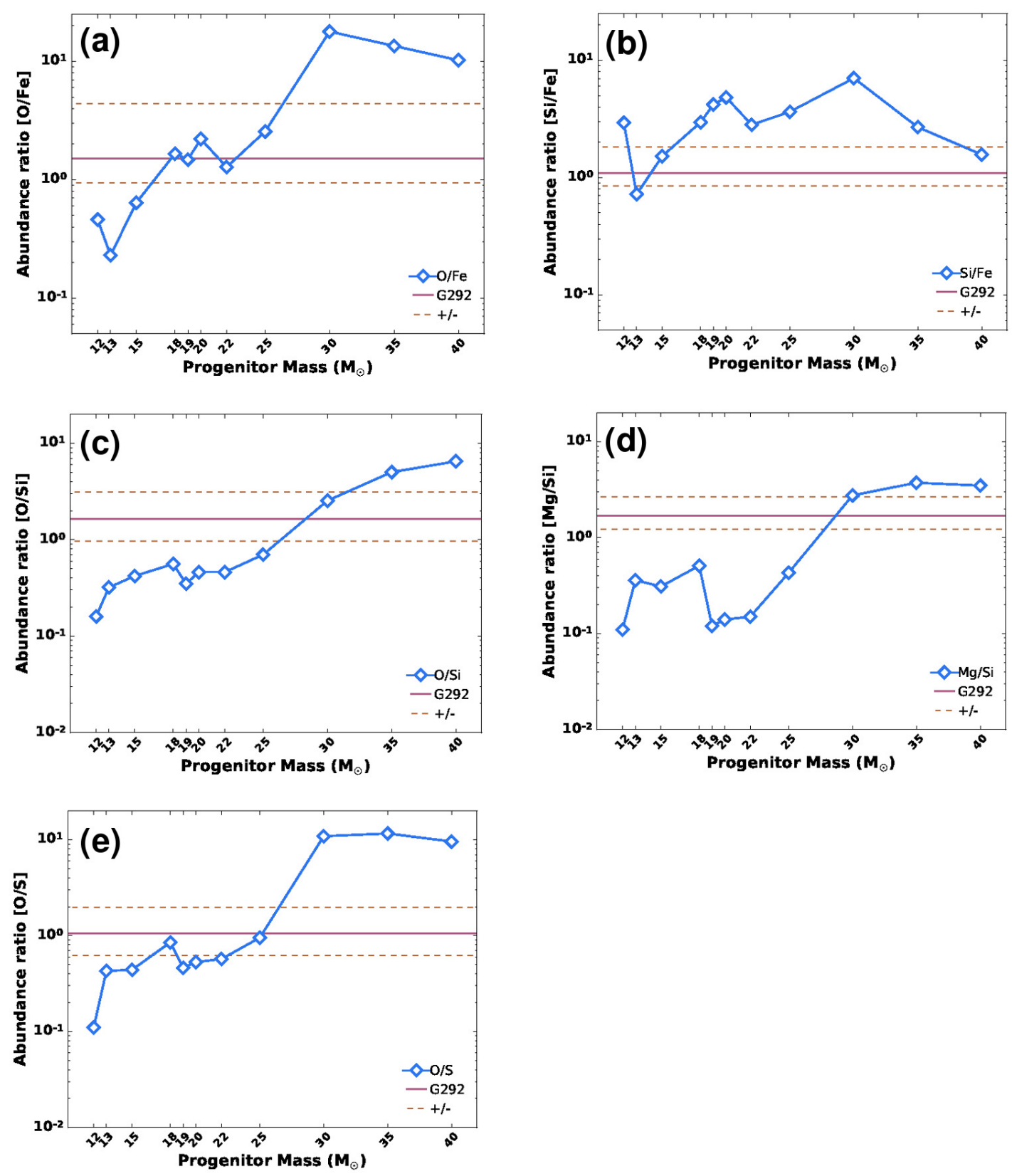

Figure 14. Average elemental abundance ratios for ejecta regions across the entire SNR (solid magenta line), compared to ratios predicted for CCSN nucleosynthesis models (blue curves, Woosley \& Weaver 1995). The plots represent the abundance ratios: (a) $\mathrm{O} / \mathrm{Fe}$, (b) $\mathrm{Si} / \mathrm{Fe}$ and (c) $\mathrm{O} / \mathrm{Si}$, (d) $\mathrm{Mg} / \mathrm{Si}$ and (e) $\mathrm{O} / \mathrm{S}$. The dashed lines represent the uncertainties in the measured abundance ratios for G292. Only regions with abundances $>1 \times$ solar, for both the elements in a given ratio, are considered when calculating these ratios. 

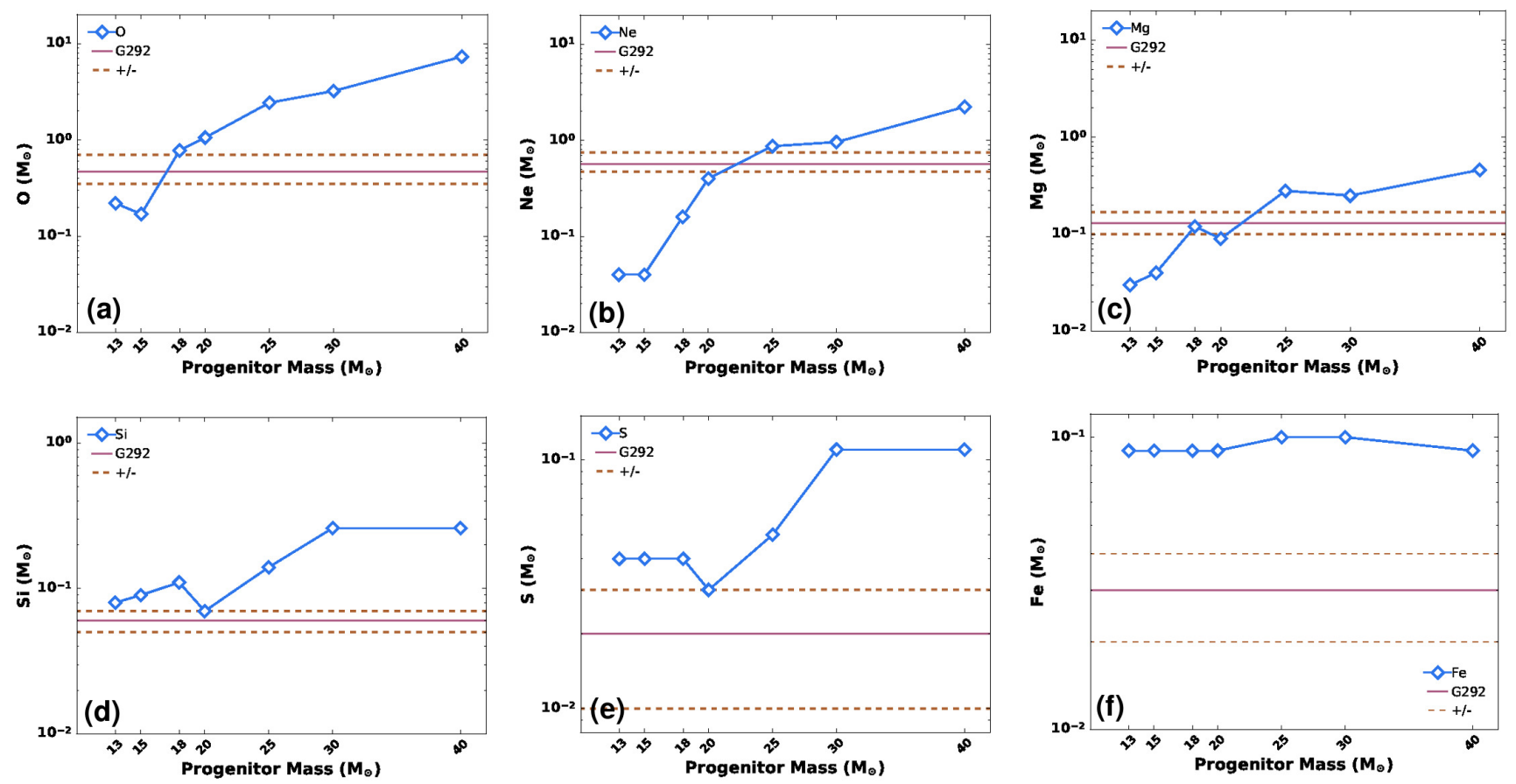

Figure 15. Elemental ejecta masses, estimated for ejecta regions across the entire SNR (solid magenta line) compared to yields predicted for CCSN nucleosynthesis models (blue curves, $Z=0.02, E=1 \times 10^{51}$ ergs, Nomoto et al. 2006). The estimated ejecta masses for G292 are in terms of $f^{1 / 2} d_{6}^{3 / 2} M_{\odot}$, where $d_{6}$ is the distance to the SNR in units of $6 \mathrm{kpc}$. The dashed lines represent the uncertainties in the estimated ejecta masses. The plotted elemental masses are (a) O, (b) Ne, (c) Mg, (d) Si, (e) $\mathrm{S}$ and (f) Fe. 